\title{
A REFINED PRECISE INTEGRATION METHOD FOR NONLINEAR DYNAMIC ANALYSIS OF STRUCTURES
}

\author{
Zhi-xia Ding ${ }^{1}$, Zuo-lei Du ${ }^{1}$, Wei Su ${ }^{2, *}$ and Yao-peng Liu ${ }^{1,3}$ \\ ${ }^{1}$ Department of Civil and Environmental Engineering, The Hong Kong Polytechnic University, Hung Hom, Kowloon, Hong Kong, China \\ ${ }^{2}$ School of Aeronautics and Astronautics, Sun Yat-sen University, Guangzhou, 510275, China \\ ${ }^{3}$ Nida Technology Co. Ltd., Hong Kong Science Park, Shatin, N.T., Hong Kong, China \\ *(Corresponding author: E-mail: suwei@mail.sysu.edu.cn)
}

\section{A B S T RA C T}

In this paper, a refined precise integration method (RPIM) is proposed for nonlinear dynamic analysis of structures. It extends the conventional precise integration method (PIM) from linear analysis to nonlinear analysis through a novel algorithm to improve the conventional Duhamel integration method for nonhomogeneous parts in nonlinear equations. In the RPIM, the stiffness matrix of the motion equation can be updated during the analysis, leading to the proposed method applicable to nonlinear structural problems. With the introduction of a new velocity vector, the original exponential matrix in PIM is reduced to a $2 \times 2$ matrix and the efficiency of RPIM is highly improved for both computation time and storage space. The analysis of stability and accuracy shows that the RPIM is unconditionally stable with highly precision. Four examples, including linear analysis of free and forced vibration and nonlinear analysis of two structures, i.e. truss and membrane, are analyzed to verify the efficiency and accuracy of the proposed RPIM.
A R T I C LE H IST TORY

$\begin{array}{ll}\text { Received: } & \text { 13 April } 2019 \\ \text { Revised: } & \text { 17 November } 2019 \\ \text { Accepted: } & \text { 3 December } 2019\end{array}$

\section{K E Y W O R D S}

Refined precise integration method; Nonlinear dynamic analysis; Stiffness matrix;

Efficiency and accuracy

\section{Introduction}

Dynamic analysis plays a critical role in the wind-induced response [1] and wave-induced response [2] in the design process of offshore steel structures, in which time is an important effect. In dynamic structural analysis, both implicit and explicit procedures are developed and adopted to deal with the equation of motion. Implicit procedures such as Newmark method [3], trapezoidal rule [4] and Wilson $\theta$-method [5], are unconditionally stable in the linear analysis but can become unstable in nonlinear analysis. Thus, equilibrium iteration is of great importance in each step [6], which involves the inverse of matrices. The explicit procedure is popular among software packages [7-8] and researchers [9-10] with the advantage of no demand on either iteration or forming tangent stiffness matrix, which is not only time consuming but also storage space consuming. Central difference method (CDM) is one such an explicit method generally adopted in many software packages, for example, the analysis software package ABAQUS [7] and OpenSees [8]. However, the CDM is conditionally stable and requires a small time increment to achieve accurate results. To avoid instability and save computation time, a new method is required to solve the differential equation for structures with amount of degrees of freedom.

The precise integration method (PIM) was firstly proposed by Zhong and Williams in 1994 [11-12] and then investigated and improved by many researchers. PIM adopts two strategies to achieve high accuracy. Firstly, PIM introduces a vector to convert the two-order differential equation to a one-order equation so that accurate results can be achieved. The vector introduced by Zhong [11-13] has been adopted by many scholars [14-15]. Gao et al. [16] also introduced a vector representing the monument of the system with specific physical meaning, so that the properties of a sparse matrix can be used to reduce the computation time and memory size. This vector was also adopted by some researchers [17]. Secondly, the exponential matrix is precisely calculated with an additional theorem [13], which is based on Taylor series introduced by Zhong [12]. It is also called the $2 \mathrm{~N}$ algorithm [13] as the time increment is further divided into $2 \mathrm{~N}$ small time domains. The value of $\mathrm{N}$ is recommended as 20 which is adequate to reach the accuracy of the computer. Another method to calculate the exponential matrix was proposed by Liu and Shen [18] based on Padé function, exhibiting better accuracy. However, the method proposed by Zhong [12] is still adopted by researchers due to its simplicity. More recently, Yue et al. [19] developed a method based on Magnus expansion to deal with exponential matrix and the high validity was approved.

One approximation in PIM is the dealing with inhomogeneous terms. Inhomogeneous terms with polynomials, exponential functions, trigonometric functions or products of these functions can be linearly interpolated with a rough approximation according to Zhong [13]. However, this method involves inverse of doubled matrix $\mathrm{H}$, formed after introducing $\mathrm{p}$ vector, which will be discussed in section 3. To avoid the disadvantage of inverse calculation of matrices, dimensional expanding method, introducing one more vector to convert nonhomogeneous terms into homogeneous ones, was proposed by Gu et al. [20]. However, this method triples the matrix which further increases computation time and memory size. Thus, the direct integration method is studied by many researchers. Lin et al. [14] decomposed the nonlinear load with Fourier expansion based on the PIM and demonstrated the accuracy of this method. Rung-Kutta method was incorporated by Zhang et al. [21] to deal with nonlinear inhomogeneous terms and the accuracy and stability of PIM were improved. Wang and Au improved the accuracy of the PIM based on Gauss quadrature method [15] and Lagrange piecewise interpolation polynomials [22]. The Simpson, Romberg and Cotes integration methods were also adopted by researchers to improve the accuracy of the PIM [23]. Tan et al. [18] proposed a method to precisely calculate the Duhamel terms based on the additional theorem, in which the nonlinear part of inhomogeneous terms is approximated by polynomials, and the linear part can then be calculated accurately. The direct integration avoids the inverse of matrix. Nevertheless, additional exponential matrices need to be calculated at integration points.

Due to the introduction of another vector to reduce the order of differential equation, the matrices in the differential equations are doubled in the PIM, leading to more computational time for the calculation of the exponential matrix. To solve this problem, amount of efforts has been made on reducing the size of matrix. Zhong et al. [24] proposed a subdomain precise time integration method so that only a small size exponential matrix is calculated each time. Wu et al. [25] also proposed a subdomain precise integration method based on the PIM for periodic structures. The whole structure is considered as a combination of several super elements and such that the computational efforts and memory size are reduced with the same accuracy. Similarly, Gao and his team [26-29] proposed subdomain PIM for periodic structures based on the parametric variational principle (PVP). With the periodic structure, only one exponential matrix is required for calculation, the efficiency is highly improved compared with calculating all the exponential matrices. Fung and Chen [30] improved computational efficiency of the PIM with Krylov subspace method and Padé approximations. Su et al. [31] computed exponential matrix with the Suzuki's decomposition based technique of the fourth order. Some efforts, different from the subdomain, to improve the efficiency of the PIM were also made by researchers. Shen et al. [19] proposed a parallel algorithm with mixed fine and coarse grain strategy to improve the efficiency of the PIM. Gao et al. [21] introduced a fast precise integration method (FPIM) considering the sparse natural of the system matrices, and the accuracy, as well as efficiency, are addressed.

Up to now, the PIM method has been investigated and developed by researchers to achieve high accuracy and increase efficiency for dynamic problems. However, there are few studies focusing on the nonlinear structural 
problems with varied stiffness matrix. In this paper, a refined precise integration method (RPIM) is proposed to deal with nonlinear dynamic problems of structures and to improve the efficiency of traditional PIM. A new vector, velocity, is introduced to simplify the calculation of the exponential matrix based on the PIM by Zhong [11] and the additional theorem of Duhamel integration by Tan [18]. After the introduction of this vector, only a $2 \times 2$ matrix is required for the calculation of the exponential matrix, with much computational time and memory size saved. In the equation of motion, the internal force, the product of varied stiffness matrix and displacement vector, are considered as an unknown variable which could be updated during the process of nonlinear analysis. Theoretically, the stability and accuracy analysis will be conducted to illustrate the stability and accuracy of the proposed method. The instability and high accuracy of the RPIM are also verified with four cases involving both linear and nonlinear dynamic analysis. Linear analysis with free vibration and forced vibration and nonlinear analysis with truss elements and membrane elements are adopted for verification of the RPIM. The accuracy of the RPIM is demonstrated by comparing with that of the CDM, and the efficiency is certified by comparison with the methods combining vectors introduced by Zhong [11] and Gao [16].

\section{Conventional central difference method}

In dynamic structural problems, the equation of motion is defined as

$$
\mathbf{M} \ddot{x}+\mathbf{C} \dot{x}+\mathbf{K} x=\mathbf{f}(x, \mathrm{t})
$$

in which $\mathrm{M}, \mathrm{C}$, and $\mathrm{K}$ are the mass, damping and stiffness matrices of the structural system respectively; $\mathbf{f}(\boldsymbol{x}, \mathrm{t})$ is the external force vector related to time and the displacements of the degrees of freedom (DOF) in each node. $\boldsymbol{x}$, $\dot{\boldsymbol{x}}$ and $\ddot{\boldsymbol{x}}$ are the vectors regard to displacements, velocities and accelerations of each DOF.

The velocity, assumed linearly distributed from time $t$ to $t+\Delta t$, and the accelerated velocity, assumed as the first-order derivative of velocity during time domain $[t-\Delta t / 2, t+\Delta t / 2]$, are respectively described as,

$$
\begin{aligned}
& \dot{\boldsymbol{x}}_{t+\Delta t / 2}=\left(\boldsymbol{x}_{t+\Delta t}-\boldsymbol{x}_{t}\right) / \Delta t \\
& \ddot{\boldsymbol{x}}_{t}=\left(\dot{\boldsymbol{x}}_{t+\Delta t / 2}-\dot{\boldsymbol{x}}_{t-\Delta t / 2}\right) / \Delta t
\end{aligned}
$$

where $\boldsymbol{x}$ is a DOF (displacement or rotation) with current time step and time increment denoted by subscript $t$ and $\Delta t$ respectively.

The central-difference integration operator is explicit in the sense that the kinematic state uses known values of $\boldsymbol{x}_{t}$ and $\ddot{\boldsymbol{x}}_{t}$ from the previous increment. The CDM is a self-starting method with the initial values of $\dot{x}_{\Delta t / 2}$ and $\dot{x}_{-\Delta t / 2}$ calculated with Eq.(4) and Eq.(5).

$$
\begin{aligned}
& \dot{\boldsymbol{x}}_{\Delta t / 2}=\dot{\boldsymbol{x}}_{0}+\ddot{\boldsymbol{x}}_{0} \Delta t / 2 \\
& \dot{\boldsymbol{x}}_{-\Delta t / 2}=\dot{\boldsymbol{x}}_{0}-\ddot{\boldsymbol{x}}_{0} \Delta t / 2
\end{aligned}
$$

During the recursion of the CDM, the inverse of matrix $\mathbf{M}+\Delta t / 2 \mathbf{C}$ is involved which might be time-consuming, especially for the case of consistent mass matrix.

\section{RPIM for dynamic structural problems}

In order to deal with nonlinear dynamic analysis, traditional PIM [11] is refined based on the additional theorem of Duhamel integration derived by Tan [32]. The essence of PIM is converting the original two-order equation of motion into a one-order differential equation by introducing a new vector. The vectors $\boldsymbol{p}$ introduced by Zhong [11] and Gao [16] are described with Eq.(6) and Eq.(7) respectively.

$$
\begin{aligned}
& \boldsymbol{p}=\mathbf{M} \dot{\boldsymbol{x}}+\mathbf{C} \boldsymbol{x} / 2, \quad \boldsymbol{u}=\left[\begin{array}{c}
\boldsymbol{x} \\
\mathbf{M} \dot{\boldsymbol{x}}+\mathbf{C} \boldsymbol{x} / 2
\end{array}\right] \\
& \boldsymbol{p}=\mathbf{M} \dot{\boldsymbol{x}}, \quad \boldsymbol{u}=\left[\begin{array}{c}
\boldsymbol{x} \\
\mathbf{M} \dot{x}
\end{array}\right]
\end{aligned}
$$

So, the motion equation can be derived as Eq.(8) and Eq.(9) respectively.

$$
\mathbf{H}=\left[\begin{array}{cc}
-\mathbf{M}^{-1} \mathbf{C} & \mathbf{M}^{-1} \\
\mathbf{M}^{-1} \mathbf{C M} / 4-\mathbf{K} & -\mathbf{C M}^{-1} / 2
\end{array}\right], \quad \dot{\boldsymbol{u}}=\mathbf{H} \boldsymbol{u}+\left[\begin{array}{c}
\mathbf{0} \\
\mathbf{f}(\boldsymbol{x}, \mathrm{t})
\end{array}\right]
$$

$$
\mathbf{H}=\left[\begin{array}{cc}
\mathbf{0} & \mathbf{M}^{-1} \\
-\mathbf{K} & -\mathbf{C M}^{-1}
\end{array}\right], \quad \dot{\boldsymbol{u}}=\mathbf{H} \boldsymbol{u}+\left[\begin{array}{c}
\mathbf{0} \\
\mathbf{f}(\boldsymbol{x}, \mathrm{t})
\end{array}\right]
$$

Obviously, the matrix formed in Eq. (9) is much simpler than that in Eq (8). The solution to these equations written by Duhamel integration as,

$$
\boldsymbol{u}(\mathrm{t})=\exp (\mathbf{H} t) \boldsymbol{x}(0)+\int_{0}^{t} \exp (\mathbf{H}(t-\tau))\left[\begin{array}{c}
\mathbf{0} \\
\mathbf{f}(\boldsymbol{x}, \mathrm{t})
\end{array}\right] d \tau
$$

Despite the accuracy of the PIM, the disadvantages of this method are obvious. The size of matrices is doubled after transformation with the introduced vector, and such that more computational efforts are demanded. Another disadvantage is that the PIM is only applicable to linear dynamic analysis since the $\mathrm{H}$ matrix should be revised and the exponential matrix is recalculated in each step if nonlinear problem is involved.

\subsection{Transformation of equation of motion}

The stiffness matrix $\mathrm{K}$ is consistent in the elastic range of structures but varies with time during the elastic-plastic range, and thus, the $\mathrm{K}$ matrix needs to be updated in the process of nonlinear analysis. In this paper, the third item $\mathrm{Kx}$ in equation of motion Eq.(1) is considered as an unknown variable, and a new $\mathbf{F}(\boldsymbol{x}, t)$ combining the external force vector $\mathbf{f}(\boldsymbol{x}, t)$ and internal force vector $\mathrm{Kx}$ is formed. Then, the equation of motion is rewritten as,

$$
\mathbf{M} \ddot{\boldsymbol{x}}+\mathbf{C} \dot{\boldsymbol{x}}=\mathbf{f}(\boldsymbol{x}, t)-\mathbf{K} \boldsymbol{x}=\mathbf{F}(\boldsymbol{x}, t)
$$

To obtain a coarser matrix with the left half being a null matrix, a new vector $\mathrm{p}$ representing the velocity is introduced.

$\boldsymbol{p}=\dot{\boldsymbol{x}}$

Writing Eq.(11) and Eq.(12) in matrix form, we have,

$\boldsymbol{u}=\left[\begin{array}{l}x \\ \dot{x}\end{array}\right], \quad \boldsymbol{u}=\mathbf{H} \dot{u}+\mathbf{F}$

in which,

$$
\begin{aligned}
& \mathbf{H}=\left[\begin{array}{cc}
\mathbf{0} & \boldsymbol{I} \\
\mathbf{0} & -\mathbf{M}^{-1} \mathbf{C}
\end{array}\right] \\
& \mathbf{F}=\left[\begin{array}{c}
\mathbf{0} \\
\mathbf{F}(\boldsymbol{x}, t)
\end{array}\right]=\left[\begin{array}{c}
\mathbf{0} \\
\mathbf{M}^{-1} \mathbf{f}(\boldsymbol{x}, t)-\mathbf{M}^{-1} \mathbf{K} \boldsymbol{x}
\end{array}\right]
\end{aligned}
$$

With a simpler transfer matrix $\mathrm{H}$, the equation of motion is converted to a differential equation of one order. The absolute solution of Eq.(13) with Duhamel integral is given mathematically as Eq.(16).

$$
\boldsymbol{u}(\mathrm{t})=\exp (\mathbf{H} t) \boldsymbol{x}(0)+\int_{0}^{t} \exp (\mathbf{H}(t-\tau)) \mathbf{F}(x, \tau) d \tau
$$

For elastic problem with constant stiffness matrix, an exact solution can be achieved with a different $\mathrm{H}$ matrix and external force $\mathbf{F}(\boldsymbol{x}, t)$ represented by Eq.(17) and Eq.(18) respectively.

$$
\mathbf{H}=\left[\begin{array}{cc}
\mathbf{0} & \boldsymbol{I} \\
-\mathbf{M}^{-1} \mathbf{K} & -\mathbf{M}^{-1} \mathbf{C}
\end{array}\right]
$$

$$
\mathbf{F}(\boldsymbol{x}, t)=\left[\begin{array}{c}
\mathbf{0} \\
\mathbf{M}^{-1} \mathbf{f}(t)
\end{array}\right]
$$

\subsection{Solution of differential equation}

For easier numerical integration, the total time is divided into a series of step time $\eta$ with time points given as,

$t_{0}=0, t_{1}=\eta, t_{2}=2 \eta, \ldots, t_{k}=k \eta, t_{k+1}=(k+1) \eta, \ldots$

The integration equation of Eq.(16) can be given as Eq.(20) with step time denoted by subscript $\mathrm{k}$. 


$$
\boldsymbol{u}_{k+1}=\boldsymbol{\Phi} \boldsymbol{u}_{k}+\int_{0}^{t} \exp \left(\mathbf{H}\left(t_{k}-\tau\right)\right) \mathbf{F}\left(\boldsymbol{x}_{k}, \tau\right) d \tau
$$

in which,

$$
\boldsymbol{\Phi}=\exp (\mathbf{H} \eta)
$$

Writing the formed 'external force' $\mathbf{F}(\boldsymbol{x}, t)$ in Polynomial form for easy integration,

$$
\mathbf{F}\left(\boldsymbol{x}_{k}, t_{k}+\tau\right)=\boldsymbol{f}_{0, k}+\tau \boldsymbol{f}_{1, k}+\cdots+\tau^{m} \boldsymbol{f}_{m, k} \quad \tau \in[0, \eta]
$$

Substituting Eq.(22) into the general solution Eq.(20), the recursion formula can be derived as,

$\boldsymbol{v}_{k+1}=\boldsymbol{\Phi} \boldsymbol{v}_{k}+\eta \boldsymbol{\Phi}_{0} \boldsymbol{f}_{0, k}+\cdots+\eta^{m+1} \boldsymbol{\Phi}_{m} \boldsymbol{f}_{m, k}$

in which,

$$
\boldsymbol{\Phi}_{m}=\frac{1}{\eta^{m+1}} \int_{0}^{\eta} \exp (\mathbf{H}(\eta-\tau)) \tau^{m} d \tau
$$

Dividing the step time $\eta$ into smaller time intervals $\tau$ with the following equation,

$$
\tau=\eta / 2^{N}
$$

$\mathrm{N}$ is an integer ideally to be large enough so that the exponential of the matrix in Eq. (21) can be calculated with Taylor series. The value of $\mathrm{N}$ was recommended as 20 by Zhong [11], which is adequate to achieve a precise value of coefficient $\boldsymbol{\Phi}$, and this value for $\mathrm{N}$ can also be adopted to calculate $\boldsymbol{\Phi}_{m}$ as described below.

To reduce the error in storing the elements in $\boldsymbol{\Phi}$ and $\boldsymbol{\Phi}_{m}$, expanding Eq.(21) and Eq.(24) with Taylor series and extracting the parts with higher orders,

$$
\begin{gathered}
\boldsymbol{\Phi}(\tau)=\mathbf{I}+\mathbf{H} \tau+\frac{(\mathbf{H} \tau)^{2}}{2 !}+\cdots+\frac{(\mathbf{H} \tau)^{l}}{l !}=\mathbf{I}+\boldsymbol{\Phi}_{a} \\
\boldsymbol{\Phi}_{m}(\tau)=\frac{1}{m+1} \mathbf{I}+\frac{1}{\eta^{m+1}} \sum_{j=1}^{\infty} \frac{(\mathbf{H} \tau)^{j}}{j !} \int_{0}^{\eta} \tau^{j}(\eta-\tau)^{m} d \tau \\
=\frac{1}{m+1} \mathbf{I}+\boldsymbol{\Phi}_{b, m}
\end{gathered}
$$

The coefficients $\boldsymbol{\Phi}_{a}$ and $\boldsymbol{\Phi}_{b}$ can be obtained by several times (20 times if $\mathrm{N}=20$ ) of iteration according to the addition theorem given by Zhong [11] and Tan [32], and the iteration equations are derived as

$$
\begin{gathered}
\boldsymbol{\Phi}_{a}=\boldsymbol{\Phi}_{a}\left(2 \mathbf{I}+\boldsymbol{\Phi}_{a}\right) \\
\boldsymbol{\Phi}_{b}=\frac{1}{2^{m+1}}\left[\boldsymbol{\Phi}_{\boldsymbol{a}}\left(\frac{1}{m+1} \mathbf{I}+\boldsymbol{\Phi}_{b, m}\right)+\mathbf{2} \boldsymbol{\Phi}_{b, m}+\mathrm{C}_{m}^{1} \boldsymbol{\Phi}_{b, m-1}\right. \\
\left.+\mathrm{C}_{m}^{2} \boldsymbol{\Phi}_{b, m-2}+\cdots\right]
\end{gathered}
$$

\subsection{Refined solution of inhomogeneous term}

The coefficients in the recursion formula Eq. (23) can be achieved from the above procedures and only the $\boldsymbol{f}_{m, k}$ expanded from 'external force' $\mathbf{F}(\boldsymbol{x}, t)$ is unsolved. Taylor series expansion can be used as described by Tan [32], but derivatives are required for relatively more accurate results which is quite difficult to obtain. The explicit method with Lagrange interpolation function is also provided in [32].

The Lagrange polynomials 'external force' $\mathbf{F}(\boldsymbol{x}, t)$ within the time period $\left[t_{k}, t_{k+1}\right]$ is given as Eq.(30), in which $\tau \in[0, \eta]$.

$$
\begin{array}{r}
\tilde{\mathbf{F}}\left(\boldsymbol{x}_{k}, t_{k}+\tau\right)=\boldsymbol{f}_{k}+\frac{11 \boldsymbol{f}_{k} / 6-3 \boldsymbol{f}_{k 1}+3 \boldsymbol{f}_{k 2} / 2-\boldsymbol{f}_{k 3} / 3}{\eta} \tau \\
+\frac{\boldsymbol{f}_{k}-5 \boldsymbol{f}_{k 1} / 2+2 \boldsymbol{f}_{k 2}-\boldsymbol{f}_{k 3} / 2}{\eta^{2}} \tau^{2} \\
+\frac{\boldsymbol{f}_{k} / 6-\boldsymbol{f}_{k 1} / 2+\boldsymbol{f}_{k 2} / 2-\boldsymbol{f}_{k 3} / 6}{\eta^{3}} \tau^{3}
\end{array}
$$

Comparing Eq.(30) with Eq.(22) leading to,

$$
\begin{aligned}
\boldsymbol{f}_{0, k} & =\boldsymbol{f}_{k}, \boldsymbol{f}_{1, k}=\frac{11 \boldsymbol{f}_{k} / 6-3 \boldsymbol{f}_{k 1}+3 \boldsymbol{f}_{k 2} / 2-\boldsymbol{f}_{k 3} / 3}{\eta} \\
\boldsymbol{f}_{2, k} & =\frac{\boldsymbol{f}_{k}-5 \boldsymbol{f}_{k 1} / 2+2 \boldsymbol{f}_{k 2}-\boldsymbol{f}_{k 3} / 2}{\eta^{2}} \\
\boldsymbol{f}_{3, k} & =\frac{\boldsymbol{f}_{k} / 6-\boldsymbol{f}_{k 1} / 2+\boldsymbol{f}_{k 2} / 2-\boldsymbol{f}_{k 3} / 6}{\eta^{3}}
\end{aligned}
$$

Substituting Eq.(31) into Eq.(22), the recursion formula is then established as,

$$
\boldsymbol{u}_{k+1}=\boldsymbol{\Phi} \boldsymbol{u}_{k}+\eta\left(\overline{\boldsymbol{\Phi}}_{0} \boldsymbol{f}_{k}+\overline{\boldsymbol{\Phi}}_{1} \boldsymbol{f}_{k-1}+\overline{\boldsymbol{\Phi}}_{2} \boldsymbol{f}_{k-2}+\overline{\boldsymbol{\Phi}}_{3} \boldsymbol{f}_{k-3}\right)
$$

in which,

$$
\begin{aligned}
& \overline{\boldsymbol{\Phi}}_{0}=\boldsymbol{\Phi}_{0}+11 \boldsymbol{\Phi}_{1} / 6+\boldsymbol{\Phi}_{2}+\boldsymbol{\Phi}_{3} / 6 \\
& \overline{\boldsymbol{\Phi}}_{1}=-3 \boldsymbol{\Phi}_{1}-5 \boldsymbol{\Phi}_{2} / 2-\boldsymbol{\Phi}_{3} / 2 \\
& \overline{\boldsymbol{\Phi}}_{2}=3 \boldsymbol{\Phi}_{1} / 2+2 \boldsymbol{\Phi}_{2}+\boldsymbol{\Phi}_{3} / 2 \\
& \overline{\boldsymbol{\Phi}}_{3}=-\boldsymbol{\Phi}_{1} / 3-\boldsymbol{\Phi}_{2} / 2-\boldsymbol{\Phi}_{3} / 6
\end{aligned}
$$

In structural engineering, the external forces are known and linearly distributed during each time increment. In commercial software package like ABAQUS [7], the amplitudes of external forces during analysis are given by tubular, periodic or other curves with specific functions. To solve structural problems, the external force can be written as a linear function in the time domain $\left[t_{k}, t_{k+1}\right]$ as,

$$
\mathbf{f}\left(t_{k}+\tau\right)=\mathbf{f}_{k}+\tau\left(\mathbf{f}_{k+1}-\mathbf{f}_{k}\right) / \eta
$$

For this external part, the exact result can be provided with Duhamel integration at $\left[t_{k}, t_{k+1}\right]$.

\subsection{Simplification of coefficient matrices}

Sparse matrices are obtained for $\boldsymbol{\Phi}$ and $\boldsymbol{\Phi}_{m}$ by introducing velocity vector, differing from the expression shown in Eq. (6) and Eq.(7), written as,

$$
\begin{gathered}
\boldsymbol{\Phi}=\left[\begin{array}{ll}
\mathbf{I} & \mathbf{T}_{12} \\
\mathbf{0} & \mathbf{T}_{22}
\end{array}\right] \\
\boldsymbol{\Phi}_{m}=\left[\begin{array}{ll}
\mathbf{I} & \boldsymbol{\Phi}_{m 1} \\
\mathbf{0} & \boldsymbol{\Phi}_{m 2}
\end{array}\right]
\end{gathered}
$$

The upper-left part of the matrices $\boldsymbol{\Phi}$ and $\boldsymbol{\Phi}_{m}$ is an identity matrix and the bottom-left part is a null matrix, leading to much simpler calculation. In structural engineering, the Rayleigh damping [33] is often adopted which is proportional to the mass matrix $\mathbf{M}$ and stiffness matrix $\mathbf{K}$, defined as,

$$
\mathbf{C}=\alpha \mathbf{M}+\beta \mathbf{K}
$$

The coefficient $\beta$ is often ignored in structural analysis and such that the matrix in Eq. (14) becomes, 


$$
\mathbf{H}=\left[\begin{array}{cc}
\mathbf{0} & \mathbf{I} \\
\mathbf{0} & -\mathbf{M}^{-1} \mathbf{C}
\end{array}\right]=\left[\begin{array}{cc}
\mathbf{0} & \mathbf{I} \\
\mathbf{0} & -\alpha \mathbf{I}
\end{array}\right]
$$

In this case, the $\mathbf{H}$ matrix is simplified as a $2 \times 2$ matrix $\mathbf{H}^{\prime}$ adopted directly in the iteration to calculate the coefficient matrix $\boldsymbol{\Phi}$,

$$
\mathbf{H}^{\prime}=\left[\begin{array}{cc}
0 & 1 \\
0 & -\alpha
\end{array}\right]
$$

Then, the coefficient matrix $\boldsymbol{\Phi}$ becomes a matrix with two different constant coefficients as described as $\boldsymbol{\Phi}^{\prime}$,

$$
\boldsymbol{\Phi}^{\prime}=\left[\begin{array}{ll}
1 & c_{1} \\
0 & c_{2}
\end{array}\right]
$$

Thus, a large amount of computation time and memory storage could be saved with respect to both the calculation of coefficients and the recursion formula to obtain the displacements and velocities, regardless of the form of mass matrix (consistent mass matrix, or lumped mass matrix). In software packages like ABAQUS [7] and OpenSees [8], the lumped mass matrix can be adopted for explicit dynamic analysis for the benefit of simplicity when calculating the inverse of mass matrix. The advantage of the reduced matrix also applies to the calculation of coefficients $\boldsymbol{\Phi}_{0}, \boldsymbol{\Phi}_{1}, \boldsymbol{\Phi}_{2}$ and $\boldsymbol{\Phi}_{3}$. After the linear operation of $\boldsymbol{\Phi}_{m}$, similar $2 \times 2$ matrix with constant values can be established as,

$$
\begin{aligned}
\overline{\boldsymbol{\Phi}}_{0}=\left[\begin{array}{ll}
\bar{\Phi}_{01} \\
\bar{\Phi}_{02}
\end{array}\right], \overline{\boldsymbol{\Phi}}_{1} & =\left[\begin{array}{ll}
\bar{\Phi}_{11} \\
\bar{\Phi}_{12}
\end{array}\right], \overline{\boldsymbol{\Phi}}_{2}=\left[\begin{array}{ll}
\bar{\Phi}_{21} \\
\bar{\Phi}_{22}
\end{array}\right], \overline{\boldsymbol{\Phi}}_{3} \\
& =\left[\begin{array}{ll}
\bar{\Phi}_{31} \\
\bar{\Phi}_{32}
\end{array}\right]
\end{aligned}
$$

It should be noted that matrix F in Eq. (15) is a vector with zero elements in the upper half, and such that the left half coefficients of matrices in Eq. (41) have no impact on the recursion results. Up to this step, all the coefficients required in the recursion formulation Eq. (41) have been converted to simple constant values, and the multiplication between matrices and vectors becomes much simpler multiplication between constant values and vectors, which greatly simplifies the calculation and saves a large amount of computation time and memory storage.

\section{The algorithm of RPIM}

For linear analysis, the stiffness matrix $\mathrm{K}$ is constant, and exact results can be derived with a different $\mathrm{H}$ matrix given in Eq. (17). This accurate method for linear analysis is defined as the accurate precise integration method (APIM) in this paper.

$$
\begin{aligned}
& \boldsymbol{x}_{k+1}=\boldsymbol{T}_{11} \boldsymbol{x}_{k}+\boldsymbol{T}_{12} \boldsymbol{v}_{k}+\eta \mathbf{M}^{-1}\left[\boldsymbol{\Phi}_{01} \boldsymbol{f}_{k}+\boldsymbol{\Phi}_{11}\left(\boldsymbol{f}_{k+1}-\boldsymbol{f}_{k}\right)\right] \\
& \boldsymbol{v}_{k+1}=\boldsymbol{T}_{21} \boldsymbol{x}_{k}+\boldsymbol{T}_{22} \boldsymbol{v}_{k}+\eta \mathbf{M}^{-1}\left[\boldsymbol{\Phi}_{02} \boldsymbol{f}_{k}+\boldsymbol{\Phi}_{12}\left(\boldsymbol{f}_{k+1}-\boldsymbol{f}_{k}\right)\right]
\end{aligned}
$$

For nonlinear analysis with variable stiffness matrix K, a refined explicit method, with the introduction of the velocity vector Eq. (12) and consideration of $\mathrm{K}$ as an unknown variable, is given in Eq. (43) based on the PIM and Duhamel integration. This method is defined as refined precise integration method (RPIM).

$$
\begin{aligned}
\boldsymbol{x}_{k+1}=\boldsymbol{x}_{k}+\boldsymbol{T}_{12} \boldsymbol{v}_{k}+ & \eta \mathbf{M}^{-1}\left[\Phi_{01} \boldsymbol{f}_{k}+\Phi_{11}\left(\boldsymbol{f}_{k+1}-\boldsymbol{f}_{k}\right)\right] \\
& +\eta \mathbf{M}^{-1}\left(\bar{\Phi}_{01} \mathbf{K}_{k} \boldsymbol{x}_{k}+\bar{\Phi}_{11} \mathbf{K}_{k-1} \boldsymbol{x}_{k-1}\right. \\
& \left.+\bar{\Phi}_{21} \mathbf{K}_{k-2} \boldsymbol{x}_{k-2}+\bar{\Phi}_{31} \mathbf{K}_{k-3} \boldsymbol{x}_{k-3}\right) \\
\boldsymbol{v}_{k+1}=\boldsymbol{T}_{22} \boldsymbol{v}_{k}+\eta \mathbf{M}^{-1}[ & \left.\Phi_{02} \boldsymbol{f}_{k}+\Phi_{12}\left(\boldsymbol{f}_{k+1}-\boldsymbol{f}_{k}\right)\right] \\
& +\eta \mathbf{M}^{-1}\left(\bar{\Phi}_{02} \mathbf{K}_{k} \boldsymbol{x}_{k}+\bar{\Phi}_{12} \mathbf{K}_{k-1} \boldsymbol{x}_{k-1}\right. \\
& \left.+\bar{\Phi}_{22} \mathbf{K}_{k-2} \boldsymbol{x}_{k-2}+\bar{\Phi}_{32} \mathbf{K}_{k-3} \boldsymbol{x}_{k-3}\right)
\end{aligned}
$$

It should be noted that the RPIM is also applicable to linear dynamic analysis with constant $\mathrm{K}$ matrix. To evaluated the efficiency of the proposed RPIM, two methods, derived from the vectors given in Eq.(6) and Eq.(7), are used as references. They are named as p-vector precise integration method (PPIM) and monument precise integration method (MPIM) respectively. The stiffness matrices K in both the PPIM and MPIM are treated as an unknown variable for better comparison with the RPIM.

To incorporate the APIM, RPIM, PPIM, and MPIM into the program for the dynamic analysis, the first step is to form $\mathrm{H}$ matrix and calculate the coefficients required in each method. Then the displacements and velocities at the next time step could be achieved with the recursion formulas in Eq. (42) or Eq. (43). It should be noted that only the APIM is self-starting while the displacements and velocities in the first three steps are required in the other three methods (e.g., RPIM, PPIM, and MPIM). In this paper, the CDM is adopted to calculate the required parameters for both linear and nonlinear problems. The algorithm flowchart of the RPIM, PPIM, and MPIM for nonlinear dynamic analysis is detailed in Fig. 1. The APIM for linear analysis is omitted in flowchart since the only difference is no requirement on the first three steps with the CDM as well as updating stiffness matrix K. For linear analysis with RPIM, PPIM, and MPIM, the step of updating K should be neglected and replaced with constant $\mathrm{K}$.

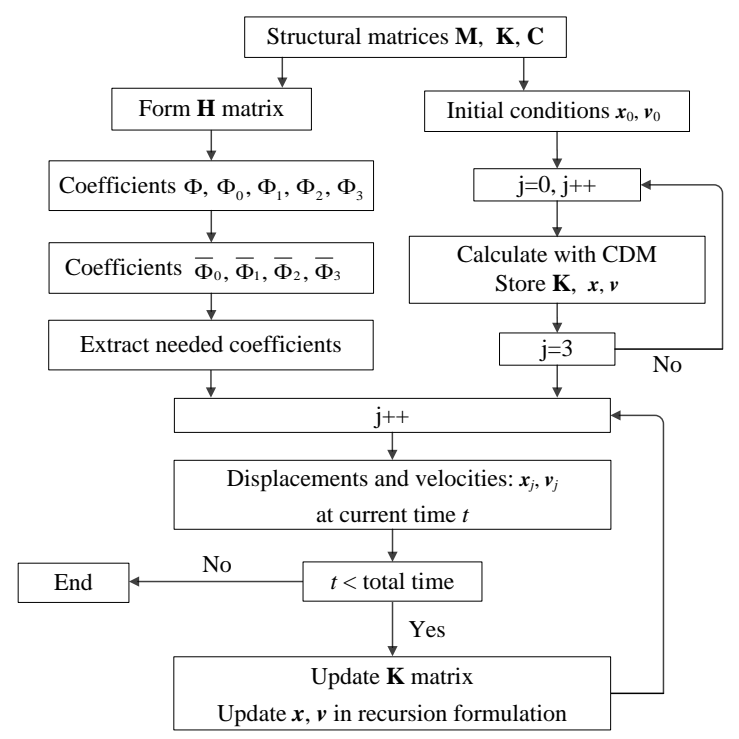

Fig. 1 Algorithm flowchart of the RPIM, PPIM, and MPIM for nonlinear dynamic analysis

\section{Stability and precision analysis}

The stability and precision are the two most important characteristics of an algorithm [34], and the performance of the RPIM will be evaluated in this section.

\subsection{Stability analysis}

The stability of a recursion formula is defined as whether the solution from recursion formula at any step time will increase infinitely with the initial conditions and rounding error in recursion. The recursion formula is unconditionally stable if the solution will not magnify without limits at any step time. To achieve unconditional stability, the coefficient $\boldsymbol{\Phi}$ must have a boundary, which means the spectral radius of $\boldsymbol{\Phi}$ cannot be greater than 1, as expressed in Eq. (44),

$$
\rho(\Phi)=\max \left|\lambda_{i}\right| \leq 1
$$

in which, $\lambda_{i}$ is the eigenvalue of $\boldsymbol{\Phi}$.

Stability analysis of the typical equation of motion with one DOF system is commonly used to verify the stability of the algorithms [35] due to the satisfaction of the mode superposition technique. The equation of motion of a structure with one DOF is defined as,

$$
\ddot{x}+2 \xi \dot{x}+\omega^{2} x=f(t)
$$

in which, $\xi$ is the damping ratio and $\omega$ is the circular frequency of the structure, with the relation presented as,

$$
\xi=\frac{c}{2 m \omega}
$$

where $\mathrm{m}$ is the mass, and $\mathrm{c}$ is the damping of the structure. The matrix $\mathrm{H}$ in Eq. (14) is then established as, 


$$
\mathbf{H}=\left[\begin{array}{cc}
0 & 1 \\
0 & -\frac{c}{m}
\end{array}\right]=\left[\begin{array}{cc}
0 & 1 \\
0 & -2 \xi \omega
\end{array}\right]
$$

As derived in Eq. (21), matrix $\boldsymbol{\Phi}$ can be obtained by substituting Eq. (28) into Eq. (26) as,

$$
\boldsymbol{\Phi}=\left[\begin{array}{cc}
1 & -\frac{f-1}{2 \xi \omega \tau} \\
0 & f
\end{array}\right]
$$

in which,

$$
f=1+(-2 \xi \omega \tau)+\frac{(-2 \xi \omega \tau)^{2}}{2 !}+\frac{(-2 \xi \omega \tau)^{3}}{3 !}+\cdots+\frac{(-2 \xi \omega \tau)^{n}}{n !}
$$

The two eigenvalues of matrix $\boldsymbol{\Phi}$ are $\lambda_{1}=1$ and $\lambda_{2}=f$ respectively. Thus, the spectral radius of $\boldsymbol{\Phi}$ is obtained as

$$
\rho(\boldsymbol{\Phi})=\max \left(\lambda_{1}, \lambda_{2}\right)=\max (1,|f|)
$$

As seen from Eq. (50), the spectral radius of $\boldsymbol{\Phi}$ depends on $|f|$. When the number of calculation term $\mathrm{n}$ tends to infinity, the following relation is obtained,

$$
|f|=e^{-2 \xi \omega \tau}<1, \quad n \rightarrow \infty
$$

When the period $\tau$ tends to be zero, the spectral radius of $\boldsymbol{\Phi}$ equals to 1, and thus the RPIM is unconditionally stable.

$$
\rho(\boldsymbol{\Phi})=\max (1,|f|)=\max (1,1)=1, \quad \tau \rightarrow 0
$$

In practice, only limited number of terms in $f$ are calculated with Eq. (49). Therefore, it is necessary to evaluate the stability with only several terms calculated in $f$. The curves of the ratio of calculation time period to basic period of structure $\tau / T$ and value of $|f|$ with different numbers of expanding terms $\mathrm{n}$ are illustrated in Fig. 2.

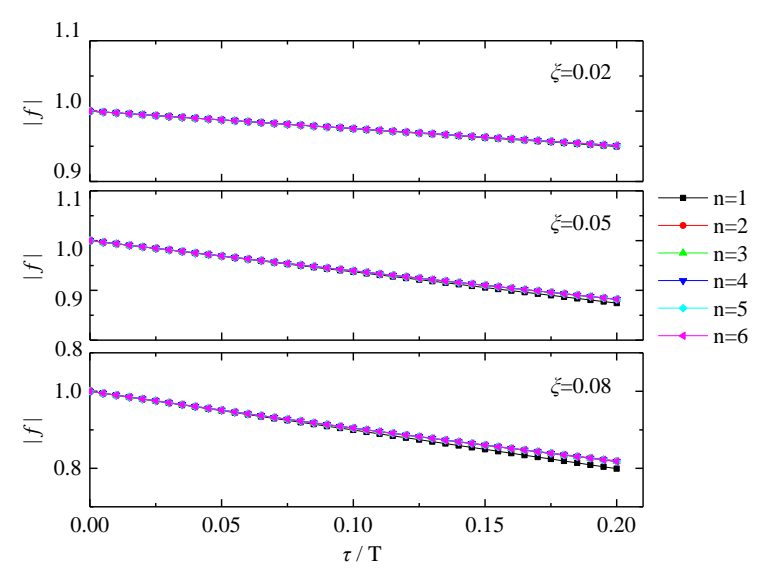

Fig. 2 Curves of $\tau / T$ and $|f|$ with different damping ratios

The curves with the damping ratio $\xi=0.02,0.05$ and 0.08 are given in Fig. 2 , within the commonly used range of structural damping ratios. According to Chinese code GB50011 [36], the damping ratio for structures is adopted as 0.05 without special instructions. Typically, $0.03 \sim 0.08$ is often adopted for reinforced concrete structures, and $0.02 \sim 0.05$ is often employed for steel structures. The period $\tau$ from Eq. (25) is very small, leading to the time ratio $\tau / T$ very close to zero. As clearly seen in Fig. 2, the values of $|f|$ under different damping ratios are less than 1, indicating the spectral radius of $\boldsymbol{\Phi}$ satisfying the spectral requirement. Thus, the unconditional spectral stability of the RPIM is demonstrated theoretically.

\subsection{Accuracy analysis}

During the derivation of recursion formula for equation of motion, the only approximation exists in the integration of inhomogeneous terms $\mathrm{Kx}$. Considering from the beginning of the derivation, the recursion formulation of Eq. (13) can be written as

$$
\boldsymbol{u}_{k+1}=\boldsymbol{\Phi} \boldsymbol{u}_{k}+\eta\left(\overline{\boldsymbol{\Phi}}_{0} \mathbf{F}_{k}+\overline{\boldsymbol{\Phi}}_{1} \mathbf{F}_{k-1}+\overline{\boldsymbol{\Phi}}_{2} \mathbf{F}_{k-2}+\overline{\boldsymbol{\Phi}}_{3} \mathbf{F}_{k-3}\right)
$$

Expanding the $\mathbf{F}_{k-1}, \mathbf{F}_{k-2}$ and $\mathbf{F}_{k-3}$ at $\mathbf{F}_{k}$ with Taylor series, then Eq.(53) can be written as,

$$
\begin{aligned}
\boldsymbol{u}_{k+1}=\boldsymbol{\Phi} \boldsymbol{u}_{k}+\eta \overline{\boldsymbol{\Phi}}_{0}( & \left.\boldsymbol{u}_{k}^{(1)}-\boldsymbol{u}_{k}\right) \\
& +\sum_{j=0}^{\infty}\left(\frac{(-\eta)^{j}}{j !} \overline{\boldsymbol{\Phi}}_{1}+\frac{(-2 \eta)^{j}}{j !} \overline{\boldsymbol{\Phi}}_{2}\right. \\
& \left.+\frac{(-3 \eta)^{j}}{j !} \overline{\boldsymbol{\Phi}}_{3}\right)\left(\boldsymbol{u}_{k}^{(j+1)}-\boldsymbol{u}_{k}^{(j)}\right)
\end{aligned}
$$

Combining the addition theorem in Eq. (28) (29) and the coefficients in Eq. (33),

$$
\begin{aligned}
\boldsymbol{u}_{k+1}=\boldsymbol{u}_{k}+\eta \boldsymbol{u}_{k}^{(1)}+ & \frac{\eta^{2}}{2} \boldsymbol{v}_{k}^{(2)}+\frac{\eta^{3}}{6} \boldsymbol{u}_{k}^{(3)}+\frac{\eta^{4}}{24} \boldsymbol{u}_{k}^{(4)} \\
& +\frac{\eta^{5}}{24}\left(-\frac{15}{2} \boldsymbol{u}_{k}^{(5)}-\frac{77}{10} \mathbf{H} \boldsymbol{u}_{k}^{(4)}+O(\eta)\right)+\cdots
\end{aligned}
$$

Thus, the RPIM with three-point Lagrange interpolation method has the precision of $O\left(\Delta t^{5}\right)$. From Eq.(54), it can be seen that the accuracy of the RPIM is closely related to the time increment $\Delta t$.

\section{Verification examples}

Several examples are analyzed to assess the accuracy and efficiency of the proposed RPIM in dealing with linear and nonlinear dynamic analysis of structures. Two linear elastic analysis cases with specific matrices, free vibration and forced vibration with constant and varied external forces, are given to provide better understanding and easier duplication of the RPIM, as well as a demonstration of application in linear dynamic analysis. In linear dynamic analysis, results from the six different methods, namely ODE45(MATLAB), APIM, CDM, RPIM, MPIM, and PPIM, are provided and discussed. The results from function ode 45 in MATLAB are set as references, in which the fourth-order and fifth-order Runge-Kutta algorithm is adopted with varied time increments, most commonly used for nonstiff problems [37]. In nonlinear dynamic analysis, only results from the last four methods are provided due to the inapplicability of the first two methods for nonlinear problems.

\subsection{Free vibration with damping}

A damped system with initial displacement at the top mass 3 is employed in this example, as shown in Fig. 3, to verify the accuracy and efficiency of the RPIM. The mass matrix $\mathrm{M}$ and stiffness matrix $\mathrm{K}$ are given directly as follows, with the damping matrix proportional to mass matrix with the relationship $\mathrm{C}=0.2 \mathrm{M}$. The initial displacement vector is given as $x_{0}=[0 ; 0 ; 5]$ and the initial velocity vector $\dot{x}_{0}=[0 ; 0 ; 0]$ is adopted.

$$
\begin{aligned}
& \mathbf{M}=\left[\begin{array}{ccc}
100 & 0 & 0 \\
0 & 100 & 0 \\
0 & 0 & 100
\end{array}\right] \\
& \mathbf{K}=10^{2}\left[\begin{array}{ccc}
200 & -100 & 0 \\
-100 & 200 & -100 \\
0 & -100 & 200
\end{array}\right]
\end{aligned}
$$

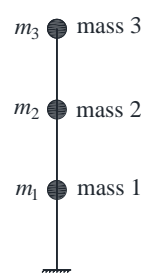

Fig. 3 Configuration of a 3-DOF system with free vibration

The displacement of mass 3 is monitored, and time-displacement curves obtained from the six methods are figured with two different time increments $\Delta t=0.005$ s (Fig. 4(a), (b)) and $\Delta t=0.02 \mathrm{~s}$ (Fig. 4(c), (d)). The MPIM and PPIM 
provide exactly the same results as RPIM, because they are essentially the same method with the only difference in introduced vector $\mathrm{p}$ as discussed in section 3. Thus, results from MPIM and PPIM are figured only when $\Delta t=0.005 \mathrm{~s}$, and are also omitted in the following verification examples to display results more clearly and intuitively.

As shown in Fig. 4(a) and (b), both the CDM and RPIM predict very accurate results at time increment $\Delta t=0.005 \mathrm{~s}$. As the time increment increases to $0.02 \mathrm{~s}$, the APIM method can still provide accurate results, the same as those from MATLAB, since there is no approximation in the APIM. Results from the RPIM are acceptable with the same variation tendency, while results from the CDM is quite unsatisfying with either too high or too low value as well as wrong variation tendency, as illustrated in Fig. 4(c) and (d).

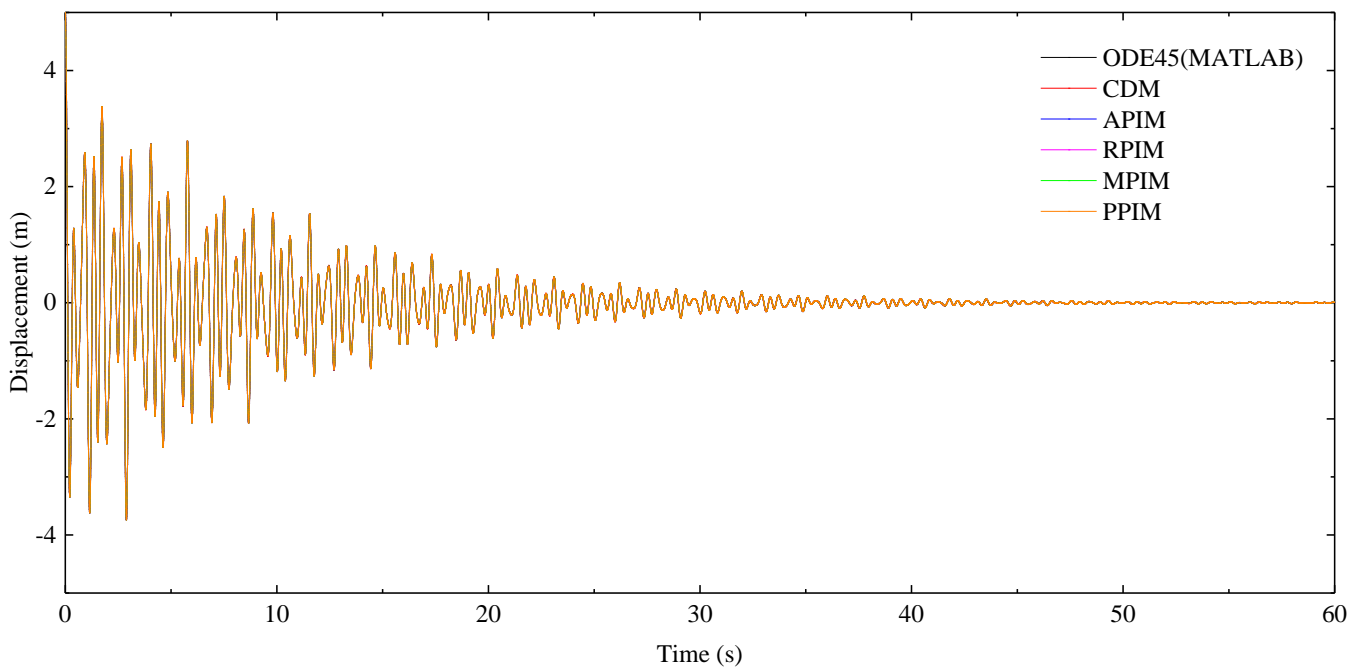

(a) Displacements in one minute with time increment $\Delta \mathrm{t}=0.005 \mathrm{~s}$

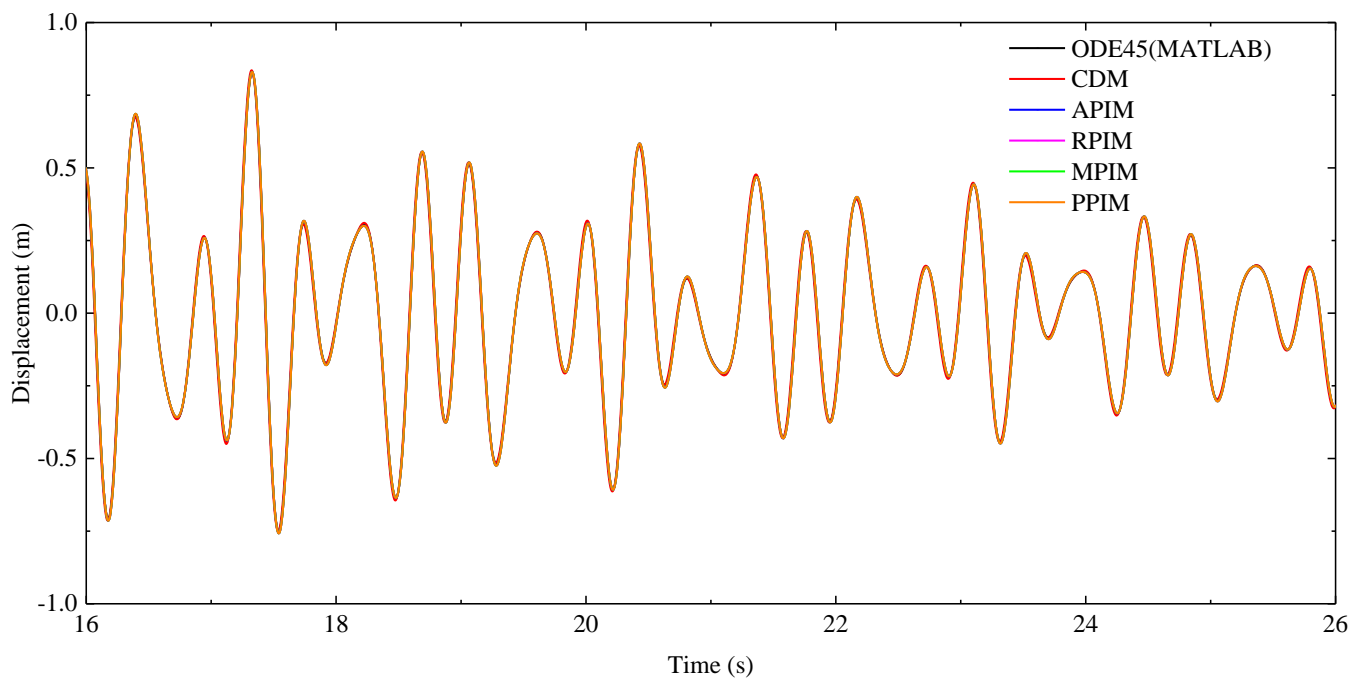

(b) Displacements from $16 \mathrm{~s}$ to $26 \mathrm{~s}$ with time increment $\Delta \mathrm{t}=0.005 \mathrm{~s}$

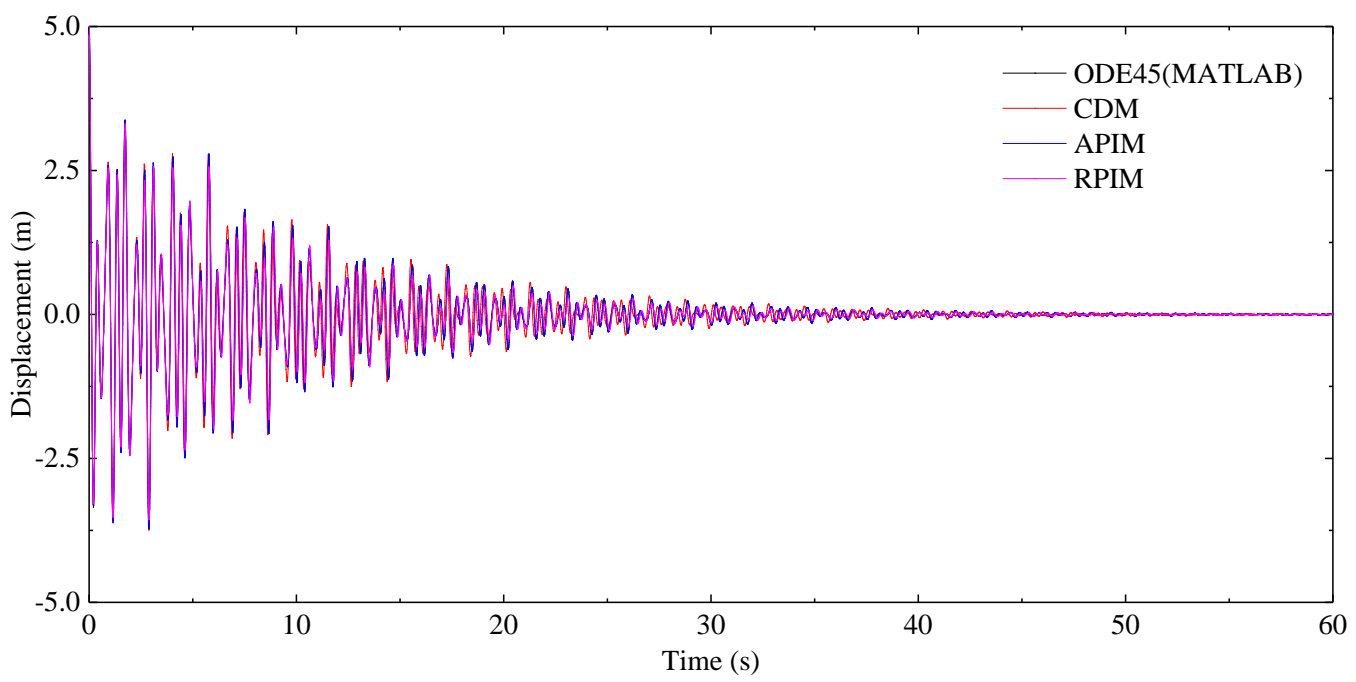

(c) Displacements in one minute with time increment $\Delta \mathrm{t}=0.02 \mathrm{~s}$ 


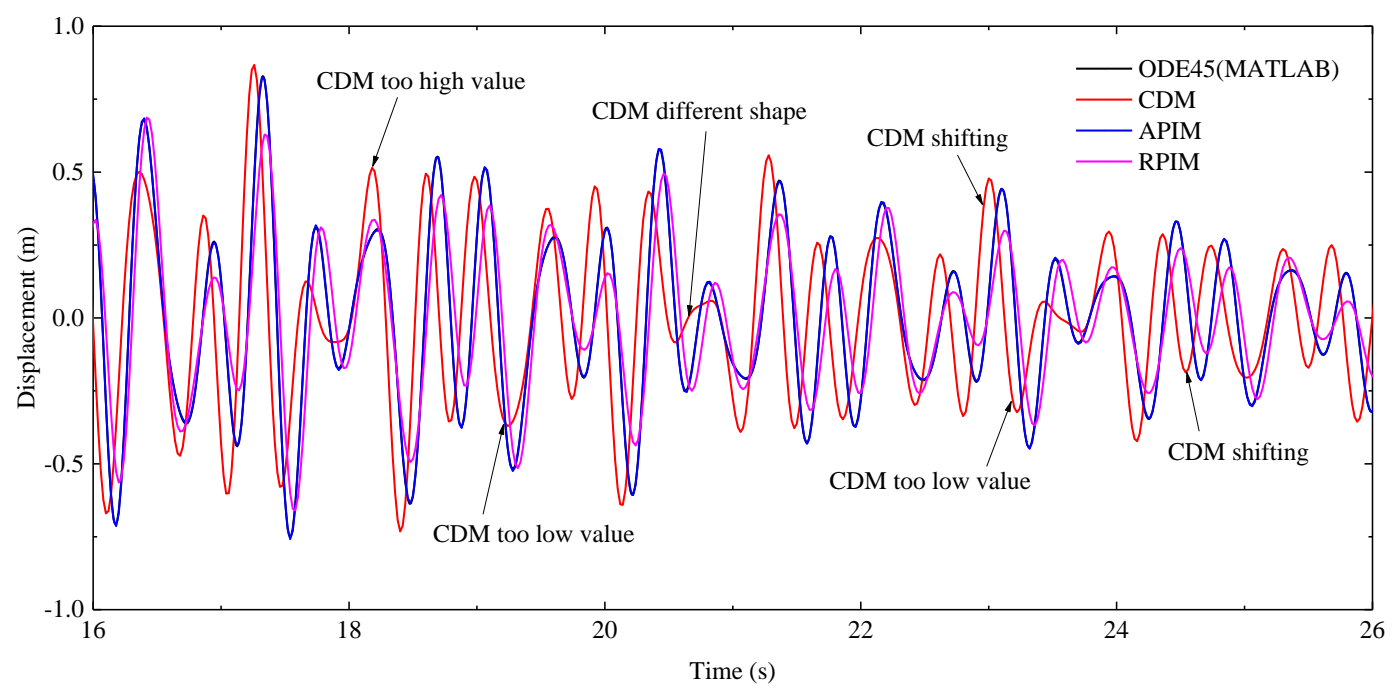

(d) Displacements from $16 \mathrm{~s}$ to $26 \mathrm{~s}$ with time increment $\Delta \mathrm{t}=0.02 \mathrm{~s}$

Fig. 4 Time-displacement curves of mass 3 during free vibration

The computational time of the RPIM, MPIM, and PPIM with different time increments are illustrated in Fig. 5, and the time reduction percentages of the RPIM compared with the PPIM are also figured. The time consumed by the MPIM is a little lower than the PPIM, but both cost more time than the RPIM. On average, about $65 \%$ computational time is reduced with the RPIM, demonstrating extraordinary efficiency of the proposed RPIM. The efficiency of RPIM is largely contributed by the reduced exponential matrix with the introduction of velocity vector.

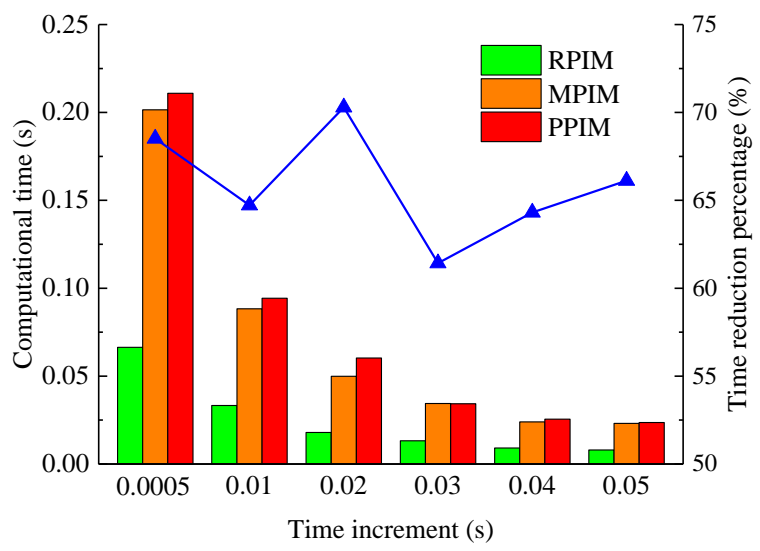

Fig. 5 Computational time and time reduction percentage during free vibration

\subsection{Forced vibration with damping}

This example is an extension of the free vibration case in section 6.1 with the same $\mathrm{M}, \mathrm{K}$ and $\mathrm{C}$ matrices. However, the different initial displacement $x_{0}=$ $[0 ; 0 ; 0]$ and constant force vector defined as follow are adopted in this case.

$$
\boldsymbol{F}(t)=[0 ; 0 ; 100]
$$

Similarly, the time-displacement curves of mass 3 obtained from the six different methods are given in Fig. 6. Exact results are provided by both the CDM and RPIM at time increment $\Delta t=0.005 \mathrm{~s}$, as shown in Fig. 6(a) and (b). As clearly seen from Fig. 6(c) and (d), results from the CDM are unsatisfying with inaccurate peak values and wrong tendency prediction at time increment $\Delta t=0.02 \mathrm{~s}$, in shape contrast to satisfying solutions provided by the proposed RPIM. Meanwhile, $35 \%$ computational time on average is economized with the RPIM when comparing with the PPIM, as illustrated in Fig. 7. It should be noted that the MPIM consumes a little fewer time than the PPIM, benefiting from simpler H matrix. Thus, superior accuracy and efficiency of RPIM are demonstrated with the ability of providing accurate results compared with the CDM and computational effort saving compared with the PPIM and MPIM.

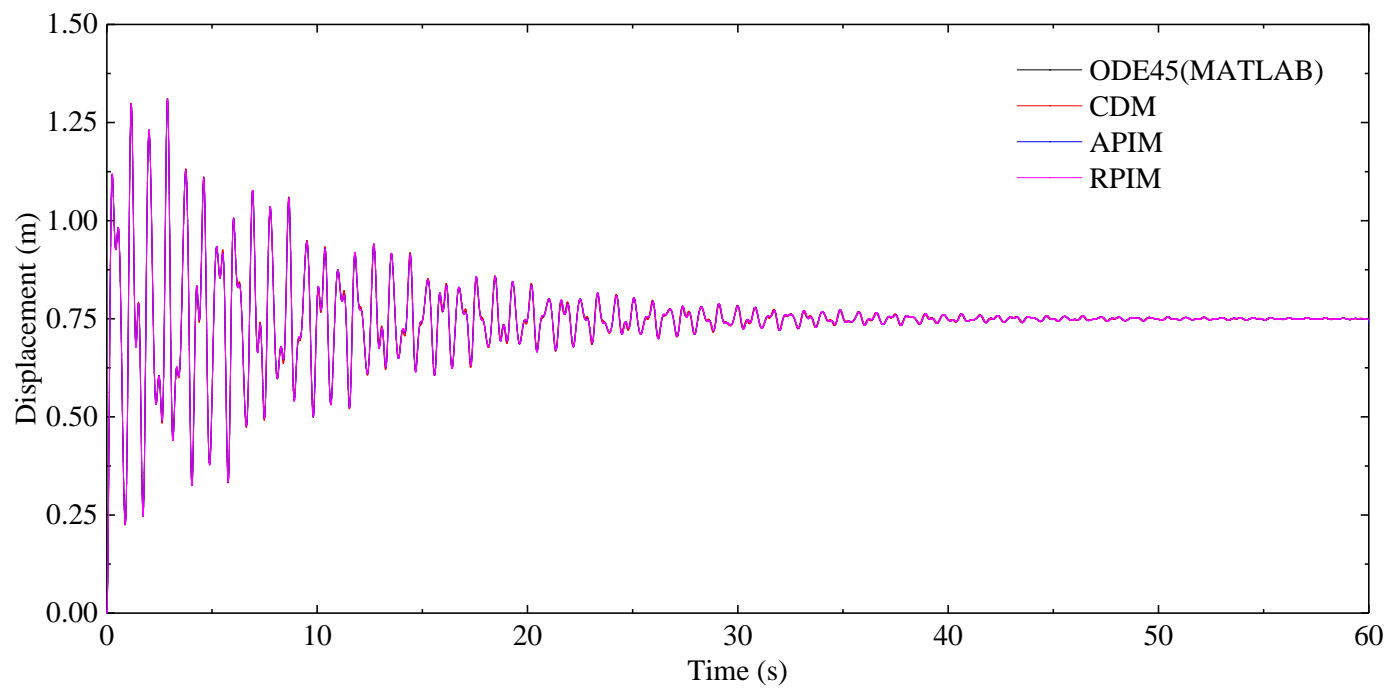

(a) Displacements in one minute with time increment $\Delta \mathrm{t}=0.005 \mathrm{~s}$ 


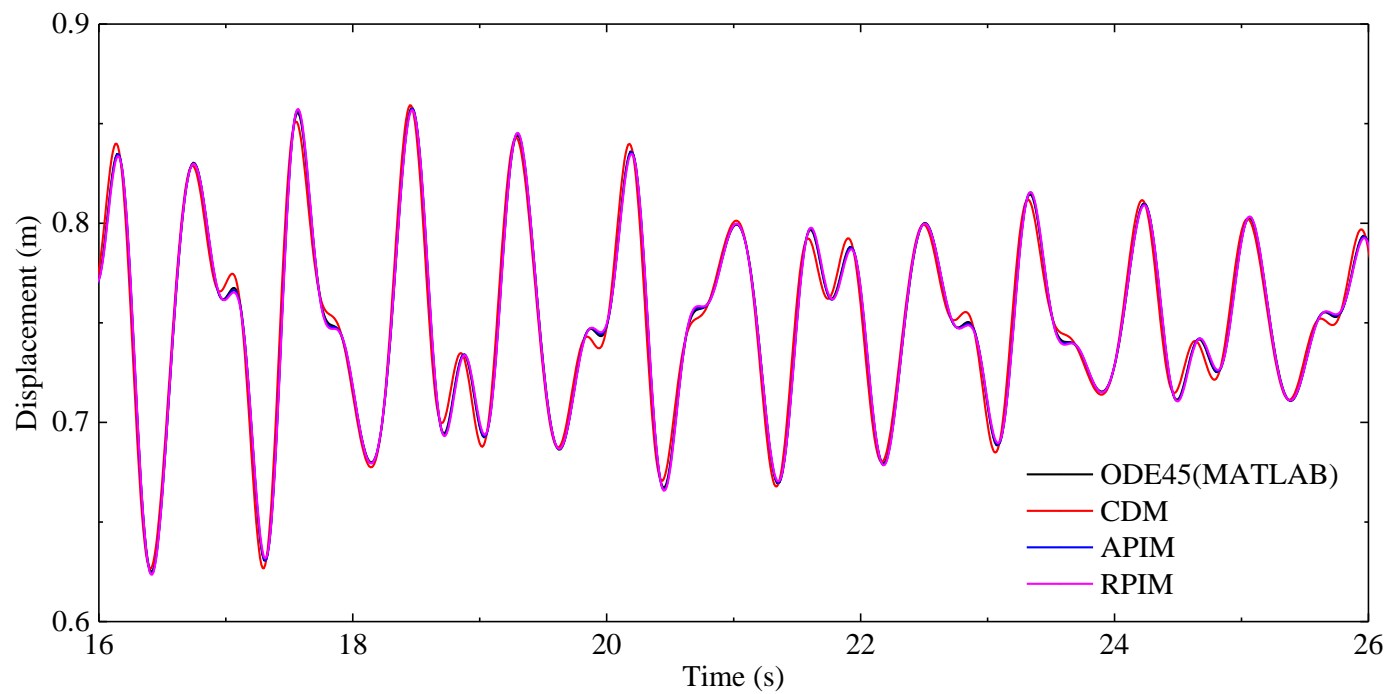

(b) Displacements from $16 \mathrm{~s}$ to $26 \mathrm{~s}$ with time increment $\Delta \mathrm{t}=0.005 \mathrm{~s}$

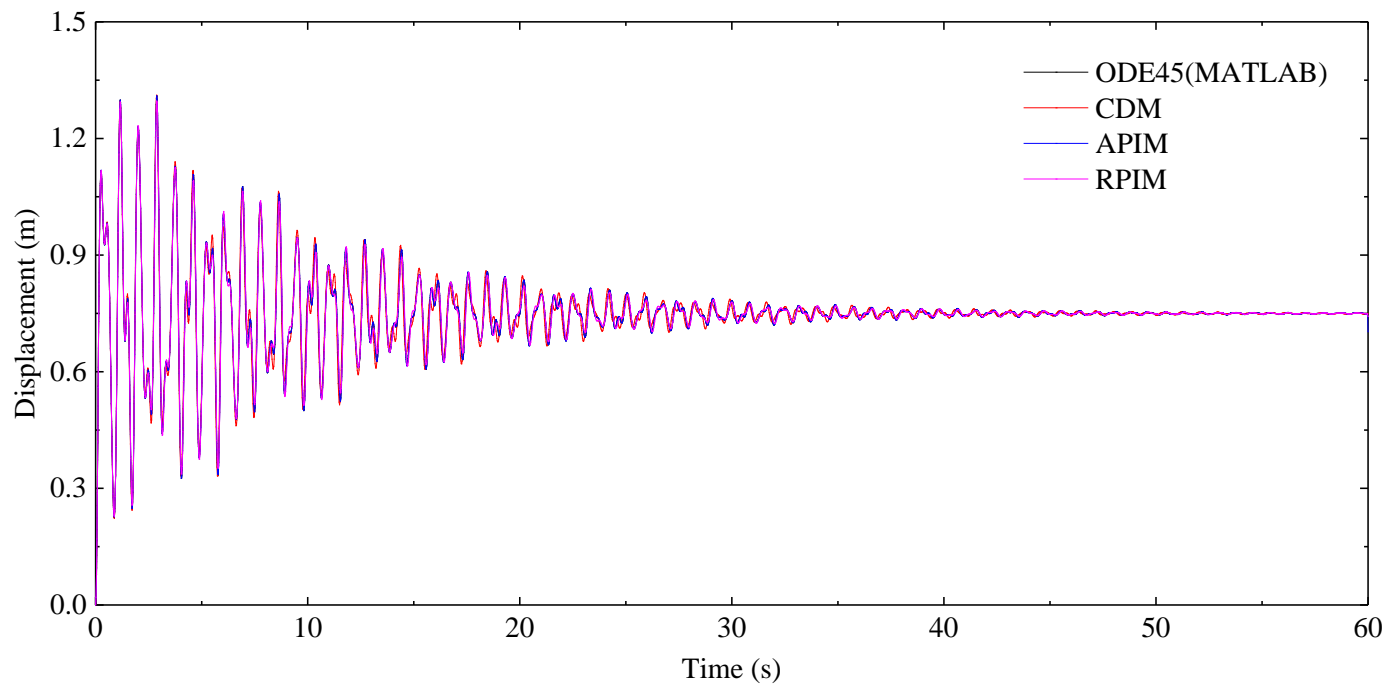

(c) Displacements in one minute with time increment $\Delta \mathrm{t}=0.02 \mathrm{~s}$

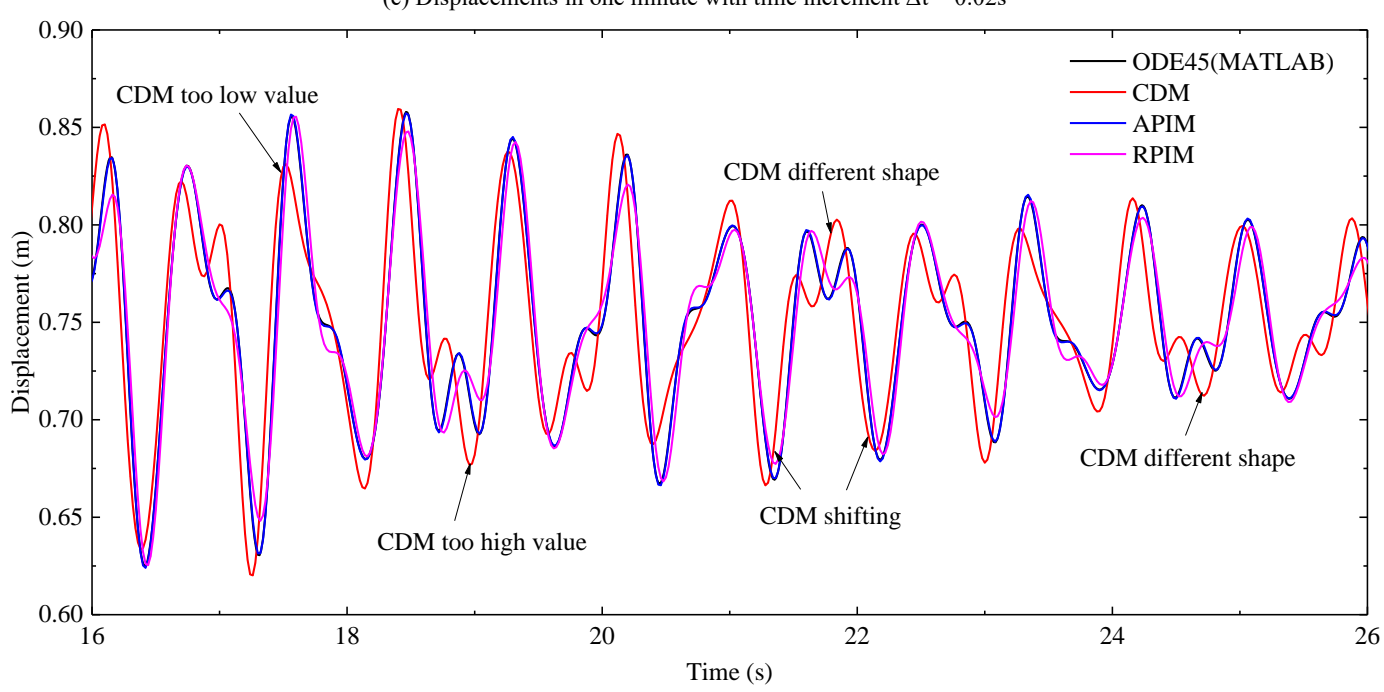

(d) Displacements from $16 \mathrm{~s}$ to $26 \mathrm{~s}$ with time increment $\Delta \mathrm{t}=0.02 \mathrm{~s}$

Fig. 6 Time-displacement curves of mass 3 during forced vibration with constant force 


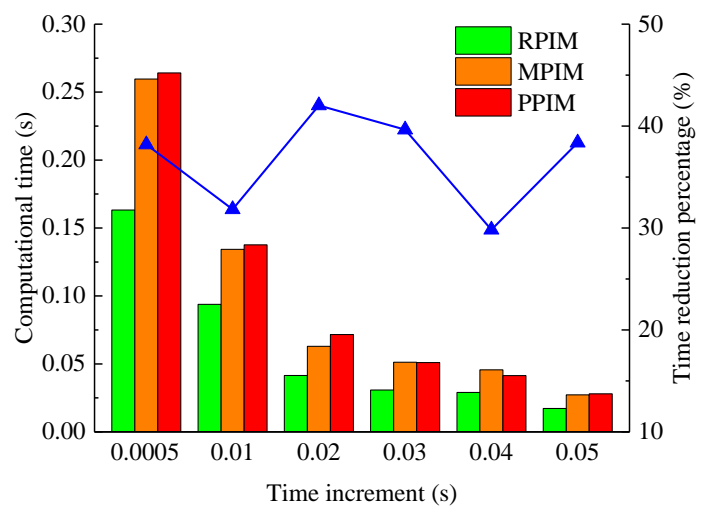

Fig. 7 Computational time and time reduction percentage during forced vibration with constant force

To assess the accuracy and efficiency of the RPIM in dealing with different forms of external loads, a varied external force with sine function is adopted written as,

$$
\boldsymbol{F}(t)=[0 ; 0 ; 100] \sin (4 \pi t)
$$

For simplicity, only results at time increment 0.02 s from different methods are given in Fig 8, Results provided by the CDM are unstable with either too high or too low values, oscillating from the accurate solutions. Nevertheless, the RPIM provides much more accurate results, as shown in Fig. 8. In addition, as shown in Fig. 9, about 35\% computational time is saved with the RPIM, similar to the percentage in the case with constant external force while less than the $65 \%$ in free vibration case. Reason for the decline of efficiency in forced vibration is that multiply operation of inversed mass matrix, and the external force is involved in each step for the RPIM, which occupying larger percentage of computational time than the calculation of exponential matrix. Nevertheless, more computational time will be saved if the external force is in the form of nodal acceleration, for example the seismic problems, since no multiply operation is required as in this case with concentrated nodal force.

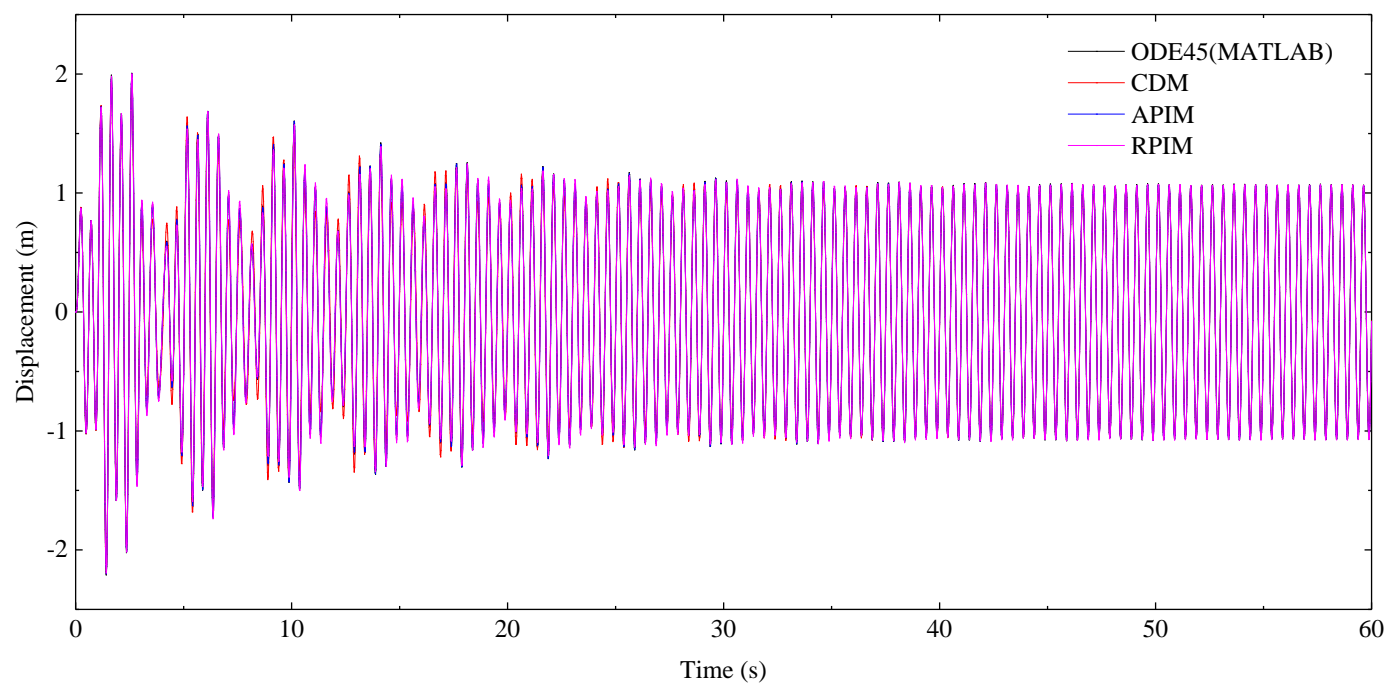

(a) Displacements in one minute with time increment $\Delta \mathrm{t}=0.02 \mathrm{~s}$

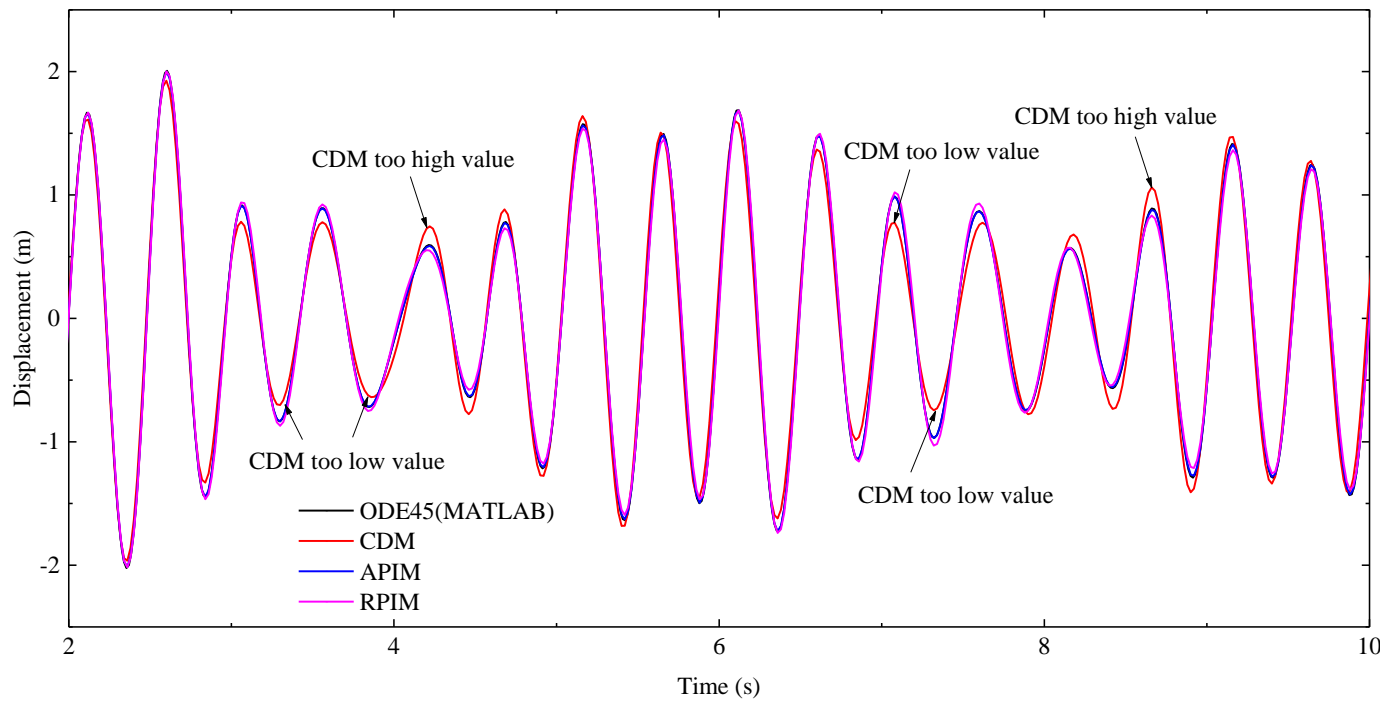

(b) Displacements from $2 \mathrm{~s}$ to $10 \mathrm{~s}$ with time increment $\Delta \mathrm{t}=0.02 \mathrm{~s}$

Fig. 8 Time-displacement curves of mass 3 during forced vibration with varied force 


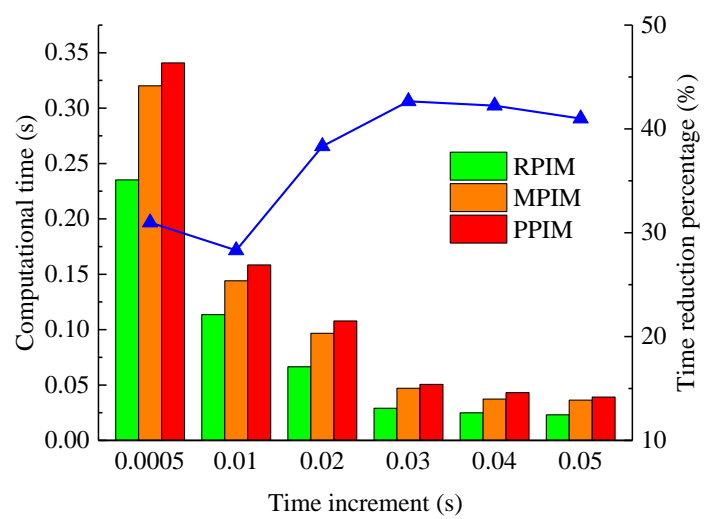

Fig. 9 Computational time and time reduction percentage during forced vibration with varied force

\subsection{Nonlinear dynamic analysis of truss}

In this example, two-dimensional Pratt truss with two DOFs (i.e., horizontal and vertical displacements) each node is adopted [38-39]. Though the RPIM has the advantage of dealing with consistent mass matrix, the lumped mass matrix is chosen for the simplicity of inverse computation, as required in ABAQUS [7] and OpenSees [8]. Only large displacement and large rotation are considered in the truss element with perfectly elastic material, ignoring material nonlinearity. There is no difference between both geometric and material nonlinearity problem, and geometric nonlinearity only problem, during the nonlinear analysis with the RPIM, since the stiffness matrix varies with time in both scenarios. Constant load $\mathrm{P}=1000 \mathrm{kN}$ is applied at node 1,2 and 3 simultaneously in ten seconds and fixed boundary condition is employed at four nodes, as illustrated in Fig. 10. Consistent section area $A=0.008 \mathrm{~m} 2$, elastic material with elastic modulus $E=2.06 \times 109 \mathrm{~N} / \mathrm{m} 2$ and density $\rho=7870 \mathrm{~kg} / \mathrm{m} 3$ are adopted in this case. The geometric information is detailed in Fig. 10

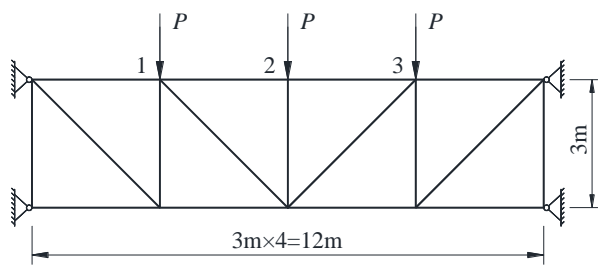

Fig. 10 Loading and boundary conditions of truss

The displacement at node 2 is monitored and curved at time increment $0.004 \mathrm{~s}$ in Fig. 11. The reference result is calculated with the CDM at a small enough time increment $0.001 \mathrm{~s}$. Results from the CDM at time increment $\Delta \mathrm{t}$ $=0.004 \mathrm{~s}$ normally shift from the accurate results as time goes by, while the RPIM provides reasonable solutions with satisfying accuracy, as shown in Fig. 11(a)(b). The accumulated error in the CDM is demonstrated well by shifting in a time-displacement curve, in great contrast to the persistent precise prediction by the RPIM. The discrepancies of the CDM and RPIM in terms of results from Reference are illustrated in Fig. 11(c). It is clearly shown that the error of the CDM is much higher than the RPIM, up to $45 \%$ at some time points. The discrepancy of results from the CDM and RPIM both decline with increasing time, with maximum error within 5\% with the RPIM and 15\% with the CDM from time $5 \mathrm{~s}$, demonstrating three times the accuracy of the RPIM as traditional CDM. Computational time is reduced by more than $15 \%$ on average with the RPIM by comparison with the PPIM, demonstrating the efficiency of the RPIM, as illustrated in Fig. 12. Time reduction percentage in this example is less than that in linear cases with forced vibration (35\%) and in free vibration case $(65 \%)$, and this is led by the updating of stiffness matrix in each step for nonlinear analysis. Nevertheless, the RPIM exhibits satisfying efficiency in nonlinear analysis. This example demonstrates the applicability of the RPIM for the analysis of steel structures like truss.

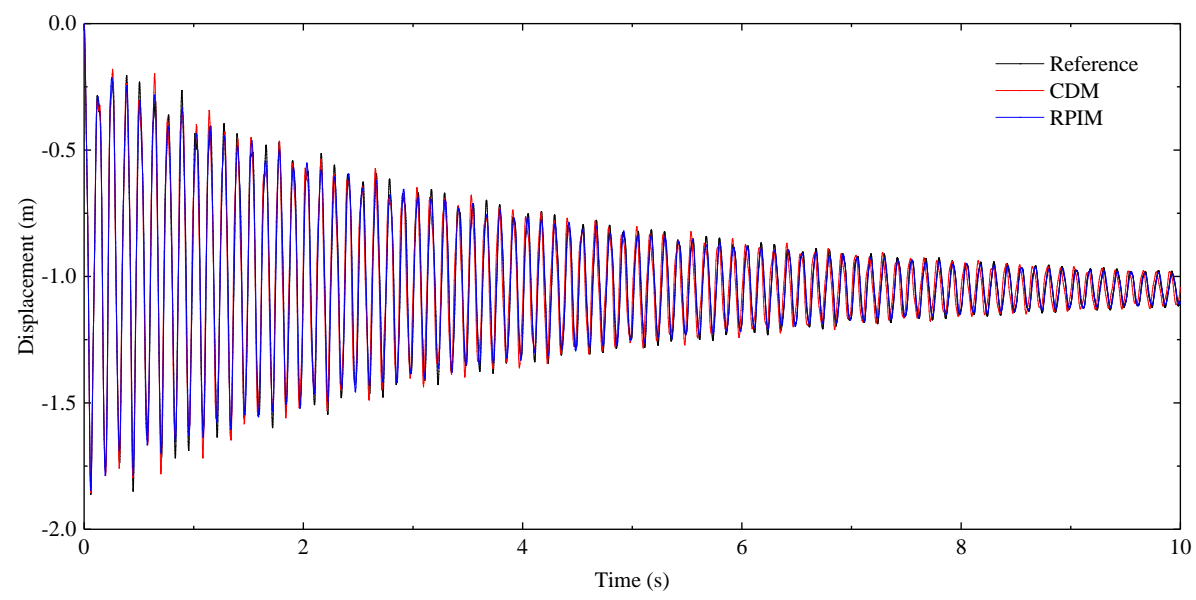

(a) Displacements in ten seconds with time increment $\Delta \mathrm{t}=0.004 \mathrm{~s}$

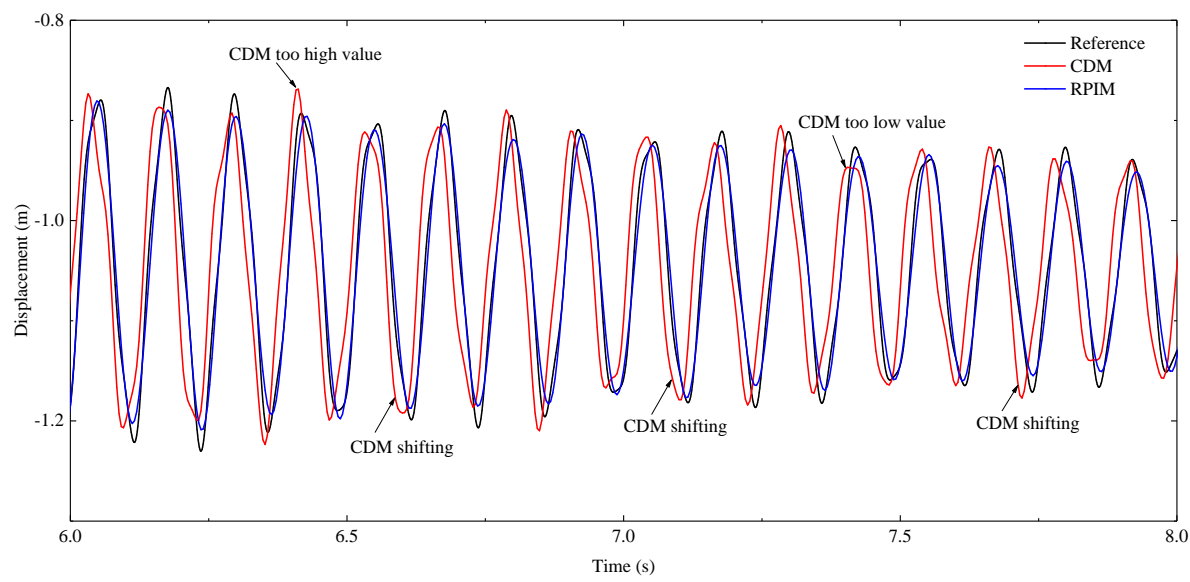

(b) Displacements from $6 \mathrm{~s}$ to $8 \mathrm{~s}$ with time increment $\Delta \mathrm{t}=0.004 \mathrm{~s}$ 


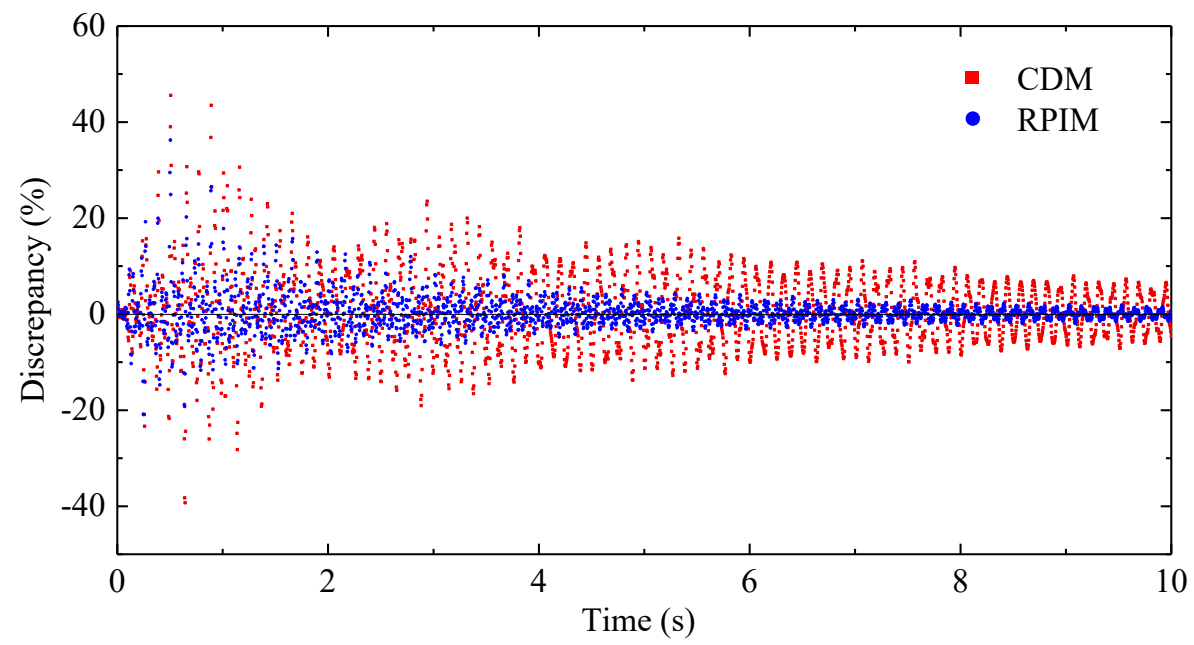

(c) Relative error of CDM and RPIM with time increment $\Delta \mathrm{t}=0.004 \mathrm{~s}$

Fig. 11 Time-displacement curves and relative error in nonlinear truss

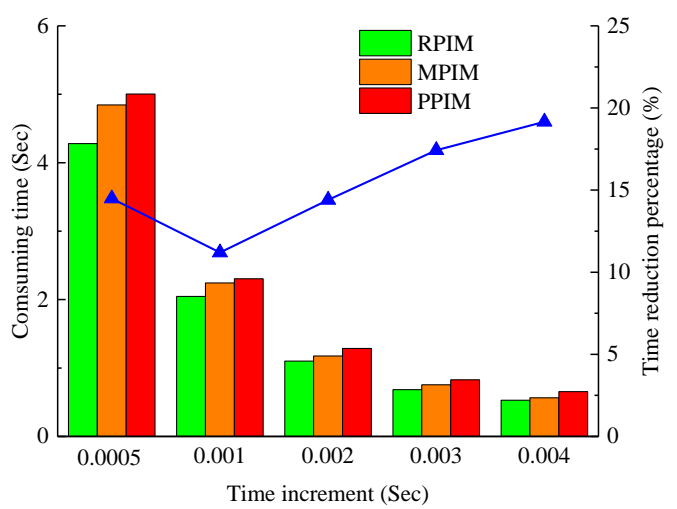

Fig. 12 Computational time and time reduction percentage in nonlinear truss

\subsection{Nonlinear analysis of membrane}

Membrane element with geometric nonlinearity only is considered in this verification example. A four-node element with two-point Gauss integration and three translation DOFs (e.g., two in-plane translation, one out-plane translation) for each node [38-39] is adopted. A $1 \mathrm{~m} \times 1 \mathrm{~m}$ square membrane with ten by ten mesh configuration is analyzed with uniform pressure $\mathrm{P}=100 \mathrm{~N} / \mathrm{m} 2$ for dynamic analysis, as illustrated in Fig. 13. The clamped boundary condition is adopted at the four sides of the membrane. Elastic material with $\mathrm{E}=2.06 \times 105 \mathrm{~N} / \mathrm{m} 2$, Poisson's ratio $\nu=0.3$, and density $\rho=7870 \mathrm{~kg} / \mathrm{m} 3$ are employed. The thickness of the membrane is consistent with $\mathrm{t}=0.005 \mathrm{~m}$.

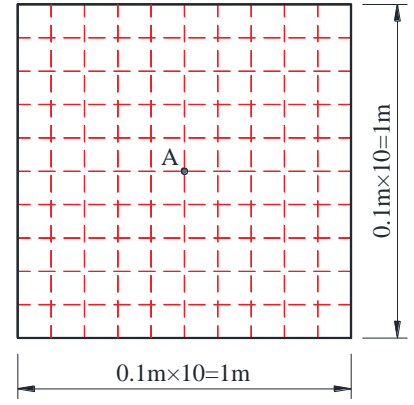

Fig. 13 Configuration of membrane

The displacements at the center point A are curved with time as illustrated in Fig. 14 with time increment $0.004 \mathrm{~s}$, and the result from the CDM with time increment $0.001 \mathrm{~s}$ is provided as reference. The overall variation tendencies of time-displacement curves obtained from both the CDM and RPIM are consistent with that from reference, as shown in Fig. 14(a). Nevertheless, different shapes and unreasonable values at the peaks are observed in the CDM from Fig. 14(b), while satisfying displacements are provided by the RPIM. The discrepancies of the CDM and RPIM with regard to reference are illustrated in Fig. 14(c). A higher error in the results by the CDM is observed with the largest error near $40 \%$ and high error remaining from time $10 \mathrm{~s}$ to $35 \mathrm{~s}$. The accuracy of the RPIM is demonstrated with the largest error near $10 \%$ in a small part of the analysis. The computational time of the RPIM is averagely $23 \%$ lower than that of the PPIM at different time increments, as illustrated in Fig. 15, and the efficiency of the RPIM is proved. Similar as in other verification examples, the computational time of the MPIM is slightly lower than that of the PPIM due to the simplicity of the introduced vector.

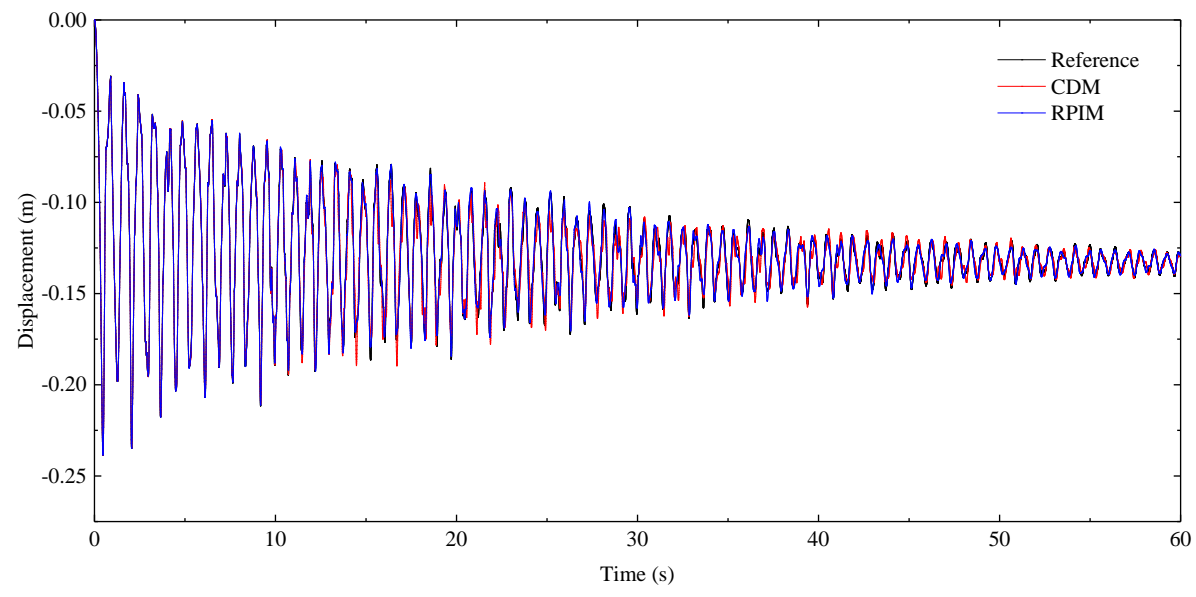

(a) Displacements in one minute with time increment $\Delta \mathrm{t}=0.004 \mathrm{~s}$ 


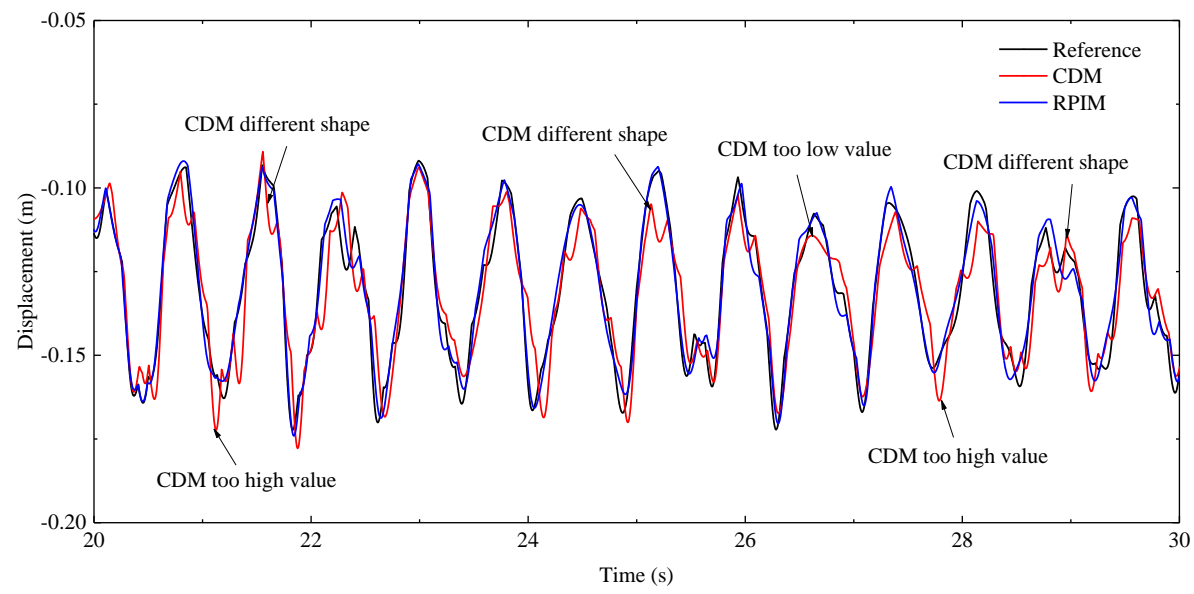

(b) Displacements from $20 \mathrm{~s}$ to $30 \mathrm{~s}$ with time increment $\Delta \mathrm{t}=0.004 \mathrm{~s}$

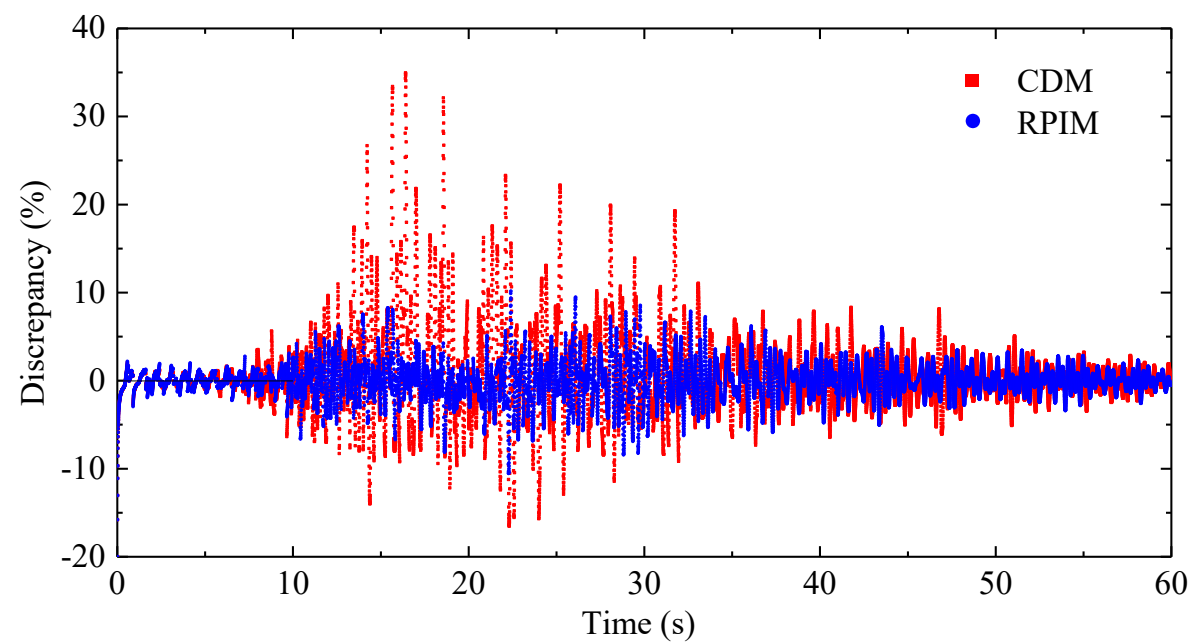

(c) Relative error of CDM and RPIM with time increment $\Delta \mathrm{t}=0.004 \mathrm{~s}$

Fig. 14 Time-displacement curves and relative error in nonlinear membrane

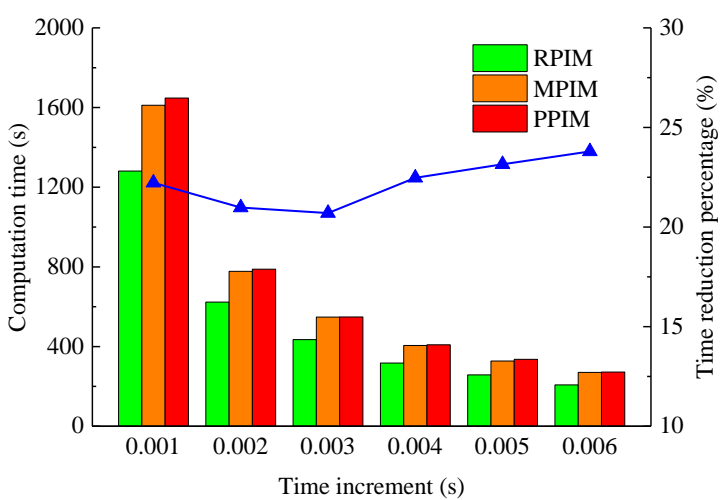

Fig. 15 Computational time and time reduction percentage in nonlinear membrane

\section{Conclusions}

In this paper, a refined precise integration method (RPIM) is proposed based on the existing precise integration method (PIM) and Duhamel integration method. It has the advantage of unconditional stability, high precision, excellent efficiency. In addition, with the transformation of equation of motion, the stiffness matrix is considered as an unknown variable which can be updated during the analysis, extending the application to nonlinear analysis. By introducing the velocity vector, the calculation of the exponential matrix is

\section{References}

[1] He, Y.J., Zhou, X.H. and Wang, H.S., "Wind-induced response analysis of the cylindrical reticulated mega-structures", Advanced Steel Construction, 12, 66-82, 2016. much simplified to the operation of a $2 \times 2$ matrix. Four typical examples containing linear and nonlinear dynamic analysis are selected to demonstrate the accuracy and efficiency of the RPIM. Several conclusions can be drawn as follows.

(1) The RPIM is unconditionally stable with fifth-order accuracy as illustrated in the stability and accuracy analysis.

(2) The calculation of the exponential matrix is much simplified with the operation of a $2 \times 2$ matrix, due to the introduction of velocity vector, and such that the efficiency of the RPIM is largely improved.

(3) The stiffness matrix is considered as a dependent variable and updated during the nonlinear analysis. Thus, the proposed RPIM is applicable to both linear and nonlinear dynamic analysis.

(4) The accuracy of the RPIM is superior to that of the CDM, since the only approximation in the RPIM exists in the internal force described with Lagrange interpolation, demonstrated well by the verification examples.

(5) No inverse of matrices is involved in the RPIM, making it applicable and efficient to deal with nonlinear dynamic analysis of large structures with adequate accuracy.

\section{Acknowledgements}

The authors are grateful for financial support from "Science and Technology Planning Project of Guangdong Province (2016A050502022) " and the Research Grant Council of the Hong Kong SAR Government on the project "Joint-based second order direct analysis for domed structures allowing for finite joint stiffness (PolyU 152039/18E)".

[2] Li Z, Ren H, Tong X, and Li H. " "A Precise Computation Method of Transient Free Surface Green Function", Ocean Engineering, 105, 318-326,2015.

[3] Newmark N.M., A Method of Computation for Structural Dynamics. Am Soc Civ Eng 1962

[4] Wilson E.L., Farhoomand I. and Bathe K.J., "Nonlinear dynamic analysis of complex structures", Earthquake Engineering Structural Dynamics, 3, 241-252, 1972. 
[5] Wilson E.L., A Computer Program for the Dynamic Stress Analysis of Underground Structure, 1968.

[6] Bathe K.J. and Baig M.M.I., "On a composite implicit time integration procedure for nonlinear dynamics", Computers and Structures, 83, 2513-2524, 2005

[7] Abaqus 6.17 Documentation. DS SIMULIA Corp, 2017.

[8]The Open System for Earthquake Engineering Simulation. Univeristy of California, Berkeley, 2000.

[9] Dokainish M.A. and Subbaraj K., "A survey of direct time-integration methods in computational structural dynamics I. Explicit methods", Computers and Structures, 32, 13711386,1989

[10] Subbaraj K. and Dokainish M.A., "A survey of direct time-integration methods in computational structural dynamics II. Implicit methods", Computers and Structures, 32, 1387-1401, 1989.

[11] Zhong W.X., "On precise time integration method for structural dynamics", Journal of Dalian University of Technology, 34, 131-136, 1994

[12] Zhong W.X. and Williams F.W., "A precise time step integration method", Proceedings of the Institution of Mechanical Engineers, Part C: Journal of Mechanical Engineering Science 208, 427-430, 1994

[13] Zhong W.X., "On precise integration method", Journal of Computational and Applied Mathematics, 163, 59-78, 2004.

[14] Lin J.H., Shen W.P. and Williams F.W., "A high precision direct integration scheme for sturctures subjected to transient dynamic loading", Computers and Structures , 56, 113-120, 1995.

[15] Wang M.F. and Au F.T.K., "Assessment and improvement of precise time step integration method", Computers and Structures, 84, 779-786, 2006.

[16] Gao Q., Wu F., Zhang H.W., Lin J.H. and Zhong W.X., "A fast precise integration method for large-scale dynamic structures", Chinese Journal of Computational Mechanics, 28, 492498, 2011.

[17] Huang C. and Fu M.H., "A modified precise integration method for long-time duration dynamic analysis", Mathematical Problems in Engineering, 2018, 1-12, 2018

[18] Liu Y. abd Shen W.P., "Adaptive algotithm of transition matrix in precise integration method", Journal of Vibration and Shock, 2, 82-85, 1995.

[19] Shen W.P., Lin J.H. and Williams F.W., "Parallel computing for the high precision direct integration method", Computer Methods in Applied Mechanics and Engineering, 126, 315331, 1995

[20] Gu Y.X., Chen B.S., Zhang H.W. and Guan Z.Q., "Precise time-integration method with dimensional expanding for structural dynamic equations", AIAA Journal, 39, 2394-2399, 2012.

[21] Gao Q., Wu F., Zhang H.W., Zhong W.X., Howson W.P. and Williams F.W., "A fast precise integration method for structural dynamics problems", Structural Engineering and Mechanics, 43, 1-13, 2012.

[22] Wang M.F. and Au F.T.K., "Precise integration methods based on Lagrange piecewise interpolation polynomials", International Journal for Numerical Methods in Engineering, 77, 998-1014, 2009.

[23] Gao Q., Tan S.J. and Zhong W.X., "A survey of the precise integration method", Scientia Sinica Technologica, 46, 1207-1218, 2016.

[24] Zhong W.X., Zhu J.N. and Zhong X.X., "On a new time integration method for solving time dependent partial differential equations", Computer Methods in Applied Mechanics and Engineering, 130, 163-178, 1996.

[25] Wu F., Gao Q. and Zhong W.X., "Subdomain Precise Integration Method for Periodic Structures", Shock and Vibration, 2014.

[26] Gao Q., Zhang H.W., Zhong W.X., Howson W.P. and Williams F.W., "An accurate and efficient method for dynamic analysis of two-dimensional periodic structures", International Journal of Applied Mechanics, 8, 2016.

[27] Gao Q. and Cui H.C. "Efficient and accurate method for 2D periodic structures based on the physical features of the transient heat conduction", International Journal of Thermal Sciences, 127, 213-231, 2018

[28] Gao Q., Yao W., Wu F., Zhang H.W., Lin J.H. and Zhong W.X., "An efficient algorithm for dynamic response of periodic structures", Chinese Journal of Theoretical and Applied Mechanics, 2011.

[29] He D.D., Gao Q. and Zhong W.X., "An efficient method for simulating the dynamic behavior of periodic structures with piecewise linearity", Nonlinear Dynamics, 94, 2018.

[30] Fung T.C. and Chen Z.L., "Krylov precise time-step integration method". International Journal for Numerical Methods in Engineering, 68, 1115-1136, 2006.

[31] Su X.X., Wang Y.S. and Zhang C.Z., "A matrix-exponential decomposition based timedomain method for calculating the defect states of scalar waves in two-dimensional periodic structures", Journal of Computational Physics, 337, 403-420, 2017.

[32] Tan S.J., Gao Q. and Zhong W.X., "Applications of Duhamel term's precise integration method in solving nonlinear differential equations", Chinese Journal of Computational Mechanics, 25, 752-758, 2010.

[33] Alipour A. and Zareian F., "Study Rayleigh Damping in Structures; Unceratinties and Treatments", The 14 ${ }^{\text {th }}$ World Conference on Earthquake Engineering, Beijing, China, 2008.

[34] Bathe K.J. and Wilson E.L., "Stability and accuracy analysis of direct integration methods", Earthquake Engineering and Structural Dynamics, 1, 3-6, 1983.

[35] Noh G. and Bathe K.J., "Further insights into an implicit time integration scheme for structural dynamics", Computers and Structures, 202, 15-24, 2018.

[36] Code for seismic design of buildings (GB50011-2010), Ministry of Housing and Urban-rural Construction of the People's Republic of China, Beijing, China, 2010.

[37] MATLAB Documentation, MathWorks, 2000.

[38] Bathe K.J., Finite element procedures in engineering analysis, Prentice-Hall, Inc, New Jersey, 1982.

[39] Ferreira A.J.M., MATLAB codes for finite element analysis: solids and structures, Springer, 2008. 